\title{
Corona und das Allgemeine Leistungsstörungsrecht
}

\author{
Prof. Dr. Thomas Riehm, Passau*
}

\section{Inhaltsübersicht}

A. Einleitung 12

B. Überblick 13

I. Vertragliche Regelungen 13

II. Gesetzlicher Rahmen 14

C. Zahlungsschwierigkeiten 16

D. Lieferschwierigkeiten beim Kaufvertrag 18

I. Geschuldete Anstrengungen des Verkäufers 19

1. Grundlagen 19

2. Grenzen der geschuldeten Anstrengungen 21

II. Allgemeine Rechtsfolgen 26

III. Rechtsfolgen bei zu vertretender Nichtleistung 26

IV. Rechtsfolgen bei nicht zu vertretender Nichtleistung 27

V. Störungen auf Seiten des Käufers 31

E. Leistungsstörungen beim Werkvertrag 32

I. Störungen auf Seiten des Unternehmers 32

1. Bauverträge nach der VOB/B 33

2. Sonstige Werkverträge 34

a) Kündigungsrecht des Bestellers ( $\$ \$ 648 \mathrm{f}$. BGB) 35

b) Rücktritt des Bestellers ( $\$ 323 \mathrm{BGB})$

c) Absolute Fixgeschäfte ( $\mathbb{S} 275$ Abs. 1, 326 BGB) 36

d) Leistungsverweigerung aus persönlichen Gründen
( $\$ 275$ Abs. $3 \mathrm{BGB})$

e) Leistungsverweigerung wegen unverhältnismäßigen Aufwandes
( $\$ 275$ Abs. 2 BGB $)$

II. Störungen auf Seiten des Bestellers $\quad 39$

F. Leistungsschwierigkeiten beim Dienstvertrag 41

I. Störungen auf Seiten des Dienstverpflichteten 41

II. Störungen auf Seiten des Dienstberechtigten 42

G. Fazit

43

* Der Autor ist Inhaber des Lehrstuhls für Deutsches und Europäisches Privatrecht, Zivilverfahrensrecht und Rechtstheorie an der Universität Passau. 


\section{A. Einleitung}

Die Covid-19-Pandemie wirft im Vertragsrecht zahlreiche drängende Fragen auf. Viele Parteien wollen jetzt wissen, was aus ihren Verträgen wird, die wegen der gegenwärtigen Pandemie nicht mehr so durchgeführt werden können, wie es ursprünglich geplant war.

Die „Corona-Krise“ wirkt sich auf verschiedenen Ebenen auf laufende Vertragsbeziehungen aus. Der Ursprung wird in vielen Fällen zunächst fehlendes Personal sein: Mitarbeiterinnen und Mitarbeiter stehen unter Quarantäne, sind selbst infiziert, werden krank, oder haben schlicht keine Betreuungsmöglichkeiten für die eigenen Kinder. Wegen des Personalengpasses können Lieferungen oder Leistungen nicht ausgeführt werden. Die hierbei auftretenden arbeitsrechtlichen Fragen sind nicht Gegenstand dieses Beitrags. ${ }^{1}$ Personalengpässe sind aber auch der Ausgangspunkt für eigene Leistungsschwierigkeiten des Arbeitgebers: Wo kein Personal ist, kann weder produziert noch geliefert noch eine Dienstleistung erbracht werden. Hinzu kommen behördliche Verbote, die sich unmittelbar oder mittelbar gegen die Leistungserbringung richten: Entweder werden Verkaufslokale direkt geschlossen oder es werden Personenansammlungen verboten, die für die Leistungserbringung unverzichtbar sind (z.B. bei Konzertveranstaltungen).

Die Folgen dieser beiden wesentlichen Störungen sind zunächst in der Liefer- bzw. Leistungskette zu spüren. ${ }^{2}$ Auf der zweiten Stufe brechen infolge des Personalengpasses die ersten Leistungen weg, sodass für die nächste Stufe der Lieferkette wichtige Vorprodukte und Vorleistungen fehlen. Dadurch kommt es zu Verzögerungen und Ausfällen in der Lieferkette, was sich immer weiter fortsetzt. ${ }^{3}$ Auf der dritten Stufe folgen aus den Liefer- und Leistungsschwierigkeiten Liquiditätsprobleme, denn wenn keine Leistungen mehr erfolgen können, fließt auch keine Gegenleistung mehr, sodass die Schuldner kein Geld mehr verdienen. Dadurch entstehen Zahlungsschwierigkeiten bis hin zur insolvenzrechtlichen Zahlungsunfähigkeit. Die insolvenzrechtlichen Sonderregelungen für die Folgen der Co-

1 S. dazu den Beitrag von S. Klawitter in diesem Band; ferner M. Fublrott, Corona und die Auswirkungen auf das Arbeitsrecht, MDR 2020, S. 540 ff.; M.-P. Weller/M. Lieberknecht/V. Habrich, Virulente Leistungsstörungen - Auswirkungen der Corona-Krise auf die Vertragsdurchführung, NJW 2020, S. 1017 (1018f.).

2 Weller/Lieberknecht/Habrich, Virulente Leistungsstörungen (Fn. 1), S. $1019 \mathrm{f}$.

3 Auch die transportrechtlichen Fragen der Lieferung sind nicht Gegenstand dieses Beitrags; s. dazu A. Maurer, in diesem Band. 
vid-19-Pandemie behandelt der Beitrag von Schmittmann ${ }^{4}$; dieser Beitrag behandelt hingegen die Konsequenzen von Zahlungsschwierigkeiten im Allgemeinen Leistungsstörungsrecht.

\section{B. Überblick}

Betrachtet man die vertragsrechtlichen Konsequenzen der Covid-19-Pandemie aus der Makroperspektive, stellt man fest, dass diese sich als externe Störung auf nahezu sämtliche Vertragsbeziehungen auswirkt. Die Kernfrage, die sich dann - zunächst noch ungeachtet der Einzelvorschriften des Zivilrechts - stellt, lautet: Wer muss dieses Risiko tragen, und wer darf es auf seinen Vertragspartner abwälzen? Diese Fragen sind im Leistungsstörungsrecht typischerweise geregelt. Dieses regelt gerade die Konsequenzen von Schwierigkeiten, die die Leistung betreffen. Manche Risiken muss der Schuldner selbst tragen: Er bekommt beispielsweise seine Gegenleistung nicht, muss vielleicht sogar Schadensersatz dafür zahlen, dass er seine Vertragspflicht nicht erfüllt. In anderen Fällen bleibt er sanktionslos und darf das Risiko der Leistungsstörung auf die Gegenseite abwälzen, bekommt also sogar seine Gegenleistung, obwohl er seine eigene Leistung nicht erbracht hat. Unter welchen Voraussetzungen welcher Fall eintritt, regelt das Leistungsstörungsrecht.

\section{Vertragliche Regelungen}

Den Ausgangspunkt für die Problembewältigung bildet allerdings zunächst die vertragliche Risikozuordnung. Manche Parteien haben in ihren Verträgen Klauseln vorgesehen, die selbst das Pandemierisiko umfassen und dessen Konsequenzen regeln. Solche Klauseln sind etwa Force Majeure-Klauseln, die typischerweise so auszulegen sein werden, dass sie auch das Pandemierisiko abdecken ${ }^{5}$ - jedenfalls, soweit dieses Risiko nicht bereits bei Vertragsschluss vorhersehbar war, was regelmäßig bei Vertrags-

4 S. J. Schmittmann, in diesem Band; ferner V. Römermann, Die Aussetzung der Insolvenzantragspflicht im COVInsAG und ihre Folgen, NJW 2020, S. $1108 \mathrm{ff}$.

5 A. Dehio/B. Rinne/C. Schmitt, Rechtliche Auswirkungen von COVID-19 im Finanzsektor, WM 2020, S. 819 (819f.); E. Wagner/R. A. Holtz/T. Dötsch, Auswirkungen von COVID-19 auf Lieferverträge, BB 2020, S. 845 (846). 
schlüssen ab Mitte März 2020 der Fall sein dürfte. ${ }^{6}$ Erfasst die Klausel das Pandemie-Risiko, weil es bei Vertragsschluss unvorhersehbar war, so gilt zwischen den Parteien das, was sie in der Force Majeure-Klausel geregelt haben. ${ }^{7}$ Beispielsweise entfällt eine Leistungspflicht, weil höhere Gewalt vorliegt, oder jemand wird von seiner Zahlungspflicht frei, oder der Vertrag wird aufgehoben. All das ist möglich, wenn die Parteien es wirksam vereinbart haben. Im Verkehr zwischen Unternehmen wird eine solche Klausel üblicherweise auch nicht an sich unangemessen benachteiligend sein, sodass eine solche Gestaltung auch in AGB möglich ist. ${ }^{8}$

Das Gleiche gilt für Material Adverse Change-Klauseln (MAC-Klauseln), die sich häufig bei Unternehmenstransaktionen, ${ }^{9}$ aber auch in Kreditverträgen ${ }^{10}$ finden. Hier ist es eine Frage der genauen Formulierung der Klausel, ob die Auswirkungen der Covid-19-Pandemie eine solche Veränderung wesentlicher Umstände darstellen; in diesem Fall gilt ebenfalls das, was in der Klausel stipuliert ist. So kann etwa vereinbart sein, dass die Transaktion aufgehoben oder angehalten wird, bis die Umstände in Nachverhandlungen o.ä. mitberücksichtigt werden.

\section{Gesetzlicher Rahmen}

Wo die Parteien allerdings keine explizite vertragliche Risikozuordnung vorgenommen haben, insbesondere das Risiko höherer Gewalt nicht ausdrücklich einer Partei zugewiesen oder anderweitig geregelt haben, gilt das gesetzliche Leistungsstörungsrecht der $\$ \mathbb{S} 275,280 \mathrm{ff}$. und $320 \mathrm{ff}$. BGB. Dieses führt indessen in der Regel zu „Alles-oder-Nichts“-Lösungen, d.h. am Ende wird entweder eine Partei von ihrer Leistungsplicht befreit oder nicht, muss für die Nichterfüllung ihrer Leistung haften oder nicht, und erhält ihre Gegenleistung oder nicht. Das allgemeine Leistungsstörungs-

6 Dehio/Rinne/Schmitt, Finanzsektor (Fn. 5), S. 820; Wagner/Holtz/Dötsch, Lieferverträge (Fn. 5), S. 848.

7 K. Bacher, Die Corona-Pandemie und allgemeinen Regeln über Leistungsstörungen, MDR 2020, S. 514 (516).

8 S. dazu Bacher, Corona-Pandemie (Fn. 7), S. 516 f.; G. Thüsing, in: F. Graf von Westphalen/G. Thüsing (Hrsg.), Vertragsrecht und AGB-Klauselwerke, 34. Aufl. 2013, Höhere Gewalt Rn. 5 ff.

9 S. dazu etwa T. Kuntz, Die Auslegung von Material Adverse Change (MAC)-Klauseln in Unternehmenskaufverträgen, DStR 2009, S. 377 ff.; C. Lange, „Material Adverse Effect” und „Material Adverse Change”-Klauseln in amerikanischen Unternehmenskaufverträgen, NZG 2005, S. 454 ff.

10 Dehio/Rinne/Schmitt, Finanzsektor (Fn. 5), S. 820. 
recht führt mit diesen Vorschriften dagegen in der Regel nicht zu vermittelnden Lösungen, die das Risiko etwa hälftig auf die Parteien verteilen.

Die Covid-19-Pandemie ist allerdings keine typische Leistungsstörung, die einzelne Schuldner betriff, die ihre Pflichten verletzt haben, weil sie etwa nicht hinreichend vorgesorgt haben. Vielmehr betriff die Pandemie alle Personen gleichermaßen; es handelt sich um eine gesamtgesellschaftliche Störung. Mithin ist auch ihre Bewältigung eine gesamtgesellschaftliche Aufgabe. Ausgehend von dieser Prämisse dürften die "Alles-oder-Nichts“Lösungen der erwähnten leistungsstörungsrechtlichen Vorschriften nicht ausreichen, um die durch die Covid-19-Pandemie ausgelösten vertragsrechtlichen Probleme zufriedenstellend zu lösen. Denn nach diesen müsste jeweils eine Partei vollständig das Risiko eines Leistungsausfalls (ihres eigenen oder desjenigen ihres Vertragspartners) tragen; eine andere Risikoverteilung als „Alles oder Nichts“ kommt grundsätzlich nicht in Betracht. Aus gesamtökonomischer Sicht werden dadurch Ergebnisse produziert, die beinahe zufällig wirken, weil die Normen, welche die Risikoverteilung im allgemeinen Leistungsstörungsrecht regeln, nicht auf derart globale und breit einwirkende Störungen ausgerichtet sind. Diese Risikoverteilung kann zu Risikoakkumulationen bei einzelnen Akteuren führen, die dann die Pandemiefolgen vollständig selbst tragen müssten, obwohl die anzuwendenden leistungsstörungsrechtlichen Vorschriften und Grundsätze (wie etwa das Beschaffungsrisiko bei Gattungsschuldnern) nicht auf Fälle wie diese zugeschnitten sind. Ein solches Ergebnis wäre allenfalls tragbar, wenn auch die staatlichen Hilfen zielgenau bei diesen Akteuren ankommen würden; dafür gibt es bisher aber keine Anhaltspunkte.

Daher ist es wichtig zu ergänzen, dass alle Ergebnisse des Allgemeinen Leistungsstörungsrechts unter dem Vorbehalt einer Korrektur wegen des Wegfalls der Geschäftsgrundlage gem. $₫ 313$ BGB stehen. Zwar ist $₫ 313$ BGB grundsätzlich insofern subsidiär gegenüber dem Allgemeinen Leistungsstörungsrecht, als die Norm nur greift, wenn keine vorrangige vertragliche oder gesetzliche Risikoverteilung besteht. ${ }^{11}$ Allerdings dürfte bei einer derart schwerwiegenden Beeinträchtigung des globalen Wirtschaftslebens, wie sie durch die Covid-19-Pandemie eingetreten ist, ein Fall vorliegen, der die gesetzliche und vertragliche Risikoverteilung in vielen Fällen überschreitet. Die betroffenen Risiken sind dann als außerhalb der gesetzlichen Risikoverteilung des allgemeinen Leistungsstörungsrechts liegend anzusehen, und werden auch von vielen vertraglichen Regelungen

11 S. Lorenz, in: H. G. Bamberger/H. Roth/W. Hau/R. Poseck (Hrsg.), Beck'scher Online-Kommentar zum BGB, 1.2.2020, $\$ 313$ Rn. 25 ff. 
(abgesehen von den bereits erwähnten Force Majeure- und MAC-Klauseln) nicht mehr erfasst sein, sodass auch keine vorrangige vertragliche Risikoverteilung anzunehmen ist. ${ }^{12}$ Alle nachfolgenden Ausführungen zum Allgemeinen Leistungsstörungsrecht stehen daher unter dem Vorbehalt, dass sich nicht aus $₫ 313$ BGB etwas anderes ergibt.

\section{Zablungsschwierigkeiten}

Der Ausgangspunkt des Allgemeinen Leistungsstörungsrechts für den Umgang mit Zahlungsschwierigkeiten ist zunächst eindeutig: Wer eine fällige und durchsetzbare Geldforderung trotz Mahnung nicht erfüllt, kommt in Verzug ( $\$ 286$ Abs. 1 BGB). Gleiches gilt für denjenigen, der einen gesetzlich oder vertraglich festgelegten Zahlungstermin nicht einhält. Das gilt auch dann, wenn die Nichtleistung darauf beruht, dass wegen Corona-bedingter Schwierigkeiten nicht gezahlt werden kann. Es gilt auch hier der Grundsatz: „Geld hat man zu haben“. ${ }^{13}$ Der Schutz des Schuldners im Fall unverschuldeter Zahlungsunfähigkeit wird erst im Insolvenzrecht oder im Vollstreckungsschutzrecht bewirkt, nicht dagegen im Allgemeinen Leistungsstörungsrecht.

Die Rechtsfolgen des Zahlungsverzuges sind bekannt: Der Geldgläubiger kann zunächst nach $\$ 320$ BGB seine Gegenleistung verweigern, das bedeutet beispielsweise beim Kaufvertrag, dass der Verkäufer nicht liefern muss, wenn der Käufer nicht bezahlt. Nach fruchtlosem Ablauf einer vom

12 S. dazu eingehend J. Prütting, in diesem Band; ferner Weller/Lieberknecht/Habrich, Virulente Leistungsstörungen (Fn.1), S. 1021 f.; a.A. offenbar S. Lorenz, in: H. Schmidt (Hrsg.), COVID-19, 2020, $\mathbb{1} 1$ Rn. 39 f.; speziell für das Mietrecht befür-

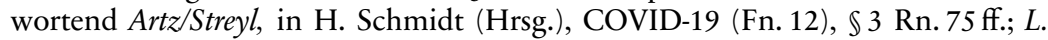
K. Kumkar/W. Voß, COVID-19 und das Institut der Geschäftsgrundlage, ZIP 2020, 893 (895 f.); M.-P. Weller/C. Thomale, Gewerbemietrecht - Mietminderung in der Corona-Krise, BB 2020, S. 962 ff.; U. Leo/E. Götz, Fälle und Lösungen zum Schicksal der Mietzahlungspflicht des Gewerberaummieters in COVID-19-Zeiten, NZM 2020, 402 (405 f.); s. zur Debatte um die Anwendbarkeit des $₫ 313$ BGB im Mietrecht neben diesen u.a. Ekkenga/Schirrmacher, Auswirkungen der COVID-19-Katastrophe auf die Zahlungspflichten gewerblicher Mieter und Pächter, NZM 2020, S. $410 \mathrm{ff}$;; A. Schall, Corona-Krise: Unmöglichkeit und Wegfall der Geschäftsgrundlage bei gewerblichen Miet- und Pachtverträgen, JZ 2020, S. 388 ff.; K. Zehelein, Infektionsschutzbedingte Schließungsanordnungen in der COVID-19-Pandemie, NZM 2020, S. 390 (397 ff.).

13 Dazu grundlegend D. Medicus, „Geld muss man haben“, AcP 188 (1988), S. 489 ff.; im vorliegenden Zusammenhang näher Lorenz (Fn. 12), $\$ 1$ Rn. 16. 
Gläubiger gesetzten Nachfrist kann dieser ferner nach $₫ 323$ Abs. 1 BGB zurücktreten. Bei Dauerschuldverhältnissen besteht im Fall von Zahlungsverzug zudem in der Regel ein Kündigungsrecht des Geldgläubigers, meist unter qualifizierten Voraussetzungen: So kann der Vermieter nach $₫ 543$ Abs. 2 S. 1 Nr. 3 BGB den Mietvertrag außerordentlich kündigen, wenn der Mieter für zwei aufeinanderfolgende Termine mit der Mietzahlung (oder eines nicht unerheblichen Teils davon, s. dazu auch $\$ 569$ Abs. 3 Nr. 1 BGB für Wohnraummietverträge) oder über einen längeren Zeitraum mit einem Betrag von mindestens zwei Monatsmieten in Verzug ist. Auch ein Darlehensgläubiger kann kündigen, wenn der Darlehensschuldner mit seiner Rückzahlung oder der Zinszahlung in Verzug gerät $(\mathbb{\$} 314$ BGB $)^{14}$ bzw. bei Verbraucherdarlehen, wenn der Darlehensnehmer mit mindestens zwei aufeinanderfolgenden Raten ganz oder teilweise oder mit einem substanziellen Teilbetrag des Darlehens in Verzug ist ( $\$ 498$ BGB). Selbst ein Strom- oder Gaslieferant kann in der Regel die Versorgung unterbrechen, wenn der Kunde mit der Begleichung seiner Rechnungen in Verzug gerät und bei wiederholtem Verzug auch den Vertrag fristlos kündigen (z.B. $\mathbb{S} 19$ Abs. 2, 21 S. 2 StromGVV, $\mathbb{S} 24$ Abs. 2, 27 S. 2 NAV bzw. S\$ 19 Abs. 2, 21 S. 2 GasGVV, $\mathbb{\$} 24$ Abs. 2, 27 S. 2 NDAV).

Zusätzlich schuldet der Geldschuldner Verzugszinsen gem. $\$ 288$ BGB: Diese betragen im Regelfall fünf Prozentpunkte über dem Basiszinssatz, bei Entgeltschulden aus Unternehmergeschäften sogar neun Prozentpunkte über dem Basiszinssatz. Hinzu kommt bei Entgeltforderungen die Kostenpauschale von $40 €$ nach $\$ 288$ Abs. 5 BGB, sowie die Pflicht zum Ersatz weiteren Verzögerungsschadens nach $\mathbb{S} 280$ Abs. 1, Abs. 2, 286 BGB, z.B. wenn der Geldgläubiger einen Anwalt beauftragen muss, um seine Forderung durchzusetzen.

Der Gesetzgeber hat allerdings erkannt, dass der Grundsatz „Geld hat man zu haben“ während der Einschränkungen durch die Covid-19-Pandemie zu unangemessenen Härten führen kann. Insbesondere die Konsequenz eines Kündigungsrechts im Mietrecht und eines Leistungsverweigerungsrechts nach $₫ 320$ BGB oder ebenfalls eines Kündigungsrechts bei Versorgungsverträgen über Energie oder Kommunikation kann für die Kunden im physischen und psychischen Sinne existenzbedrohend werden. Daher wurden im neuen Art. $240 \$ 1$ EGBGB Sonderregeln ${ }^{15}$ geschaffen,

14 S. dazu C. A. Weber, in: beck-online.Großkommentar zum Zivilrecht, 15.2.2020, $\$ 490 \mathrm{Rn} .149 \mathrm{ff}$.

15 Zum gesamten Art. 240 EGBGB s. den Überblick bei M. Schmidt-Kessel/C. Möllnitz, Coronavertragsrecht - Sonderregeln für Verbraucher und Kleinstunternehmen, NJW 2020, S. 1103 ff. 
die die Versorgung von Verbrauchern und Kleinstunternehmern mit lebensnotwendigen Leistungen auch bei Corona-bedingten Zahlungsschwierigkeiten sicherstellen sollen (Verbraucher-Moratorium). ${ }^{16}$ Das hierdurch begründete Leistungsverweigerungsrecht schließt es aus, dass die Geldgläubiger (also die Versorgungsunternehmen) die Versorgung nach $\$ 320$ BGB verweigern oder gar wegen Zahlungsverzugs einstellen dürfen. ${ }^{17}$

Zudem schützt Art. $240 \$ 2$ EGBGB Mieter und Pächter vor Zahlungsverzugskündigungen ${ }^{18}$ und Art. $240 \rrbracket 3$ EGBGB Darlehensnehmer bei Verbraucherdarlehen vor Kündigungen wegen Zahlungsverzugs oder wegen des Wertverlusts von Sicherheiten. ${ }^{19}$ Diese Sonderregelungen betreffen jedoch bei weitem nicht alle Fälle Corona-bedingter Leistungsschwierigkeiten, sodass das allgemeine Leistungsstörungsrecht nach wie vor von Bedeutung ist.

\section{Lieferschwierigkeiten beim Kaufvertrag}

Beim Kaufvertrag können sich Corona-bedingte Einschränkungen zunächst in Gestalt von Personalengpässen beim Hersteller niederschlagen, falls der Verkäufer zugleich Hersteller ist, also auf der ersten Handelsstufe. Dann kann der Hersteller evtl. nicht so viele Waren produzieren, wie er vertraglich versprochen hatte. Zudem können Corona-bedingt evtl. notwendige Vorprodukte nicht beschafft werden, etwa weil die Logistikkette durch Grenzschließungen oder Betriebsschließungen bei Vorlieferanten unterbrochen wird, sodass die Produktion aus diesem Grund ins Stocken

16 S. dazu A.-M. Kaulbach/B. Scholl, in diesem Band, sowie T. Rüfner, JZ 2020,

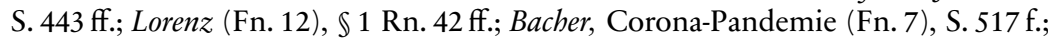
C. Möllnitz/M. Schmidt-Kessel, in: W. Uhlenbruck (Hrsg.), Insolvenzordnung, 15. Aufl. 2020, Art. 240 \$ $\$ 1$ 1-4 EGBGB Rn. 7 ff.

17 Möllnitz/Schmidt-Kessel (Fn. 16), Art. 240 \$S 1-4 EGBGB Rn. 79.

18 S. dazu J. Brinkmann, in diesem Band, sowie M. Artz/J. Brinkmann/D. Pielsticker, Wohnraummietrecht in Zeiten der Corona-Pandemie, MDR 2020, S. 527 ff.; Möllnitz/Schmidt-Kessel (Fn. 16), Art. 240 \$S 1-4 EGBGB Rn. 94 ff.; S. Sittner, Mietrechtspraxis unter Covid-19, NJW 2020, S. 1169 ff.; zu Zweifeln an der Verfassungsmäßigkeit der Regelung s. K.-M. Uth/H. Barthen, Verfassungswidrigkeit der Kündigungsbeschränkung im „COVID-19-Gesetz“, NZM 2020, S. 385 ff.

19 S. dazu Kaulbach/Scholl, in diesem Band, sowie T. B. Lühmann, Das Moratorium im Darlehensrecht anlässlich der Covid-19-Pandemie, NJW 2020, S. 1321 ff.; E. Meier/M. Kirschhöfer, Auswirkungen der Corona-Gesetzgebung auf laufende Darlehensverträge, BB 2020, S. 967 ff.; Möllnitz/Schmidt-Kessel (Fn. 16), Art. 240 \$S 1-4 EGBGB Rn. $129 \mathrm{ff}$. 
gerät. Denkbar ist auch, dass der Betrieb des Herstellers unmittelbar aufgrund einer behördlichen Anordnung geschlossen und die Produktion aus diesem Grund stillgelegt wird. All diese Gründe führen dann auf der nächsten Handelsstufe zu Lieferschwierigkeiten, weil Händler nicht hinreichend mit Waren beliefert werden, um die eigenen Lieferverpflichtungen gegenüber ihren Abnehmern erfüllen zu können.

\section{Geschuldete Anstrengungen des Verkäufers}

Die erste Frage in diesen Fällen lautet, welche Anstrengungen der Verkäufer unternehmen muss, um seiner Lieferpflicht noch nachkommen zu können.

\section{Grundlagen}

Der dogmatische Ausgangspunkt hierfür ist, dass es sich bei der Lieferpflicht nach $\$ 433$ Abs. 1 S. 1 BGB um eine erfolgsbezogene Leistungspflicht handelt, d.h. der Verkäufer schuldet die Übergabe und Übereignung der Kaufsache, nicht nur das Bemühen darum. Er muss also grundsätzlich alles unternehmen, um diese Leistungspflicht zu erfüllen. Das bedeutet, er muss selbstverständlich sein Lager leerräumen und alle Lagerbestände ausliefern. Er muss, wenn er selbst Händler und nicht (nur) Produzent ist, sogar am Markt Waren zukaufen - etwa bei anderen Großhändlern, wenn bei diesen noch welche verfügbar sind.

Das gilt allerdings nicht für einen selbst produzierenden Verkäufer, der sich von vornherein nur verpflichtet hat, selbst produzierte Waren zu verkaufen (beschränkte Gattungsschuld ${ }^{20}$ ); dieser muss nicht etwa bei der Konkurrenz vergleichbare Waren erwerben, sondern schuldet von vornherein nur Bestände aus seiner Eigenproduktion, soweit diese noch auf Lager sind oder produziert werden können. Allerdings schuldet der Produzent seinen Käufern im Hinblick auf bestehende Lieferverpflichtungen immerhin die optimale Organisation seines Produktionsbetriebs, d.h. er muss diesen im Rahmen des Möglichen und Zumutbaren so organisieren, dass eine Erfüllung der bereits eingegangenen Lieferverpflichtungen möglich bleibt. Dazu muss er ggfs. Personal umschichten und andere organisatori-

20 Vgl. OLG Hamburg OLGE 33, 208 f.; weitere Beispiele bei Riehm, in: beck-online.Großkommentar zum Zivilrecht (Fn. 14), 1.2.2020, \275 Rn. 57.1. 
sche Maßnahmen treffen, um den Produktionsbetrieb noch aufrecht zu erhalten, solange das möglich ist.

Sofern nur noch begrenzte Bestände vorhanden sind, die nicht für die Erfüllung sämtlicher zuvor eingegangener Leistungspflichten genügen, verlangt die herrschende Meinung sowohl vom Händler als auch vom Produzenten, dass diese Bestände proportional unter allen Käufern aufzuteilen sind. Je nach dem Volumen der jeweiligen zuvor abgeschlossenen Kaufverträge soll jeder Käufer einen entsprechenden Anteil erhalten - ähnlich wie bei der Verteilung einer Insolvenzmasse unter gleichrangigen Gläubigern. ${ }^{21}$ Nach der Gegenauffassung, die auch hier vertreten wird, wird dem Verkäufer zugestanden, nach freiem Ermessen zu entscheiden, welchen bzw. welche Käufer er befriedigen möchte. ${ }^{22}$ Dieses Ermessen wird er typischerweise danach ausüben, welche Kundenbeziehung ihm am wichtigsten ist, welcher Käufer die schärfsten Konsequenzen androht oder schlicht, wer als Erstes klagt und vollstreckt. Diese Auffassung bezieht sich letztlich auf den Prioritätsgrundsatz der Einzelzwangsvollstreckung, der außerhalb von Insolvenzverfahren Geltung beansprucht. Es wäre zudem im etwaigen Lieferprozess und auch in einem späteren Schadensersatzprozess nicht realistisch zu erwarten, dass der Verkäufer vorträgt (und das Gericht prüft), welche Kaufverträge insgesamt mit welchem Volumen bestanden, welche Waren insgesamt vorhanden waren, und wie eine proportionale Aufteilung ausgesehen hätte. Dabei handelt es sich nicht zuletzt um Geschäftsgeheimnisse des Verkäufers. Und schließlich öffnet die h.M. dem Verkäufer Tür und Tor für die Schutzbehauptung im Falle einer Teilleistungsstörung, dass er die fehlenden Waren wegen eines konkurrierenden obligatorischen Anspruchs eines Dritten nicht liefern durfte. Nach hier vertretener

21 RG Recht 1918, 152 (Nr. 306); RGZ 84, 125 (128 f.); OLG Augsburg JW 1919, 736 (737); OLG Hamburg OLGE 32, 314 (315) (jeweils bei Rohstoffmangel im ersten Weltkrieg); H. P. Westermann, Drittinteressen und öffentliches Wohl als Elemente der Bewertung privater Rechtsverhältnisse, AcP 208 (2008), S. 141 (168 f.); J. Neuner, Der Prioritätsgrundsatz im Privatrecht, AcP 203 (2003), S. 46 (57 f.); U. Huber, Leistungsstörungen, Band 1, 1999, \$2 24 IV 3 (S. 601 ff.); B. Gsell, Beschaffungsnotwendigkeit und Leistungspflicht, 1998, S. 159 ff.; W. Ernst, Die Konkretisierung in der Lehre vom Gattungskauf, in: W. Schön (Hrsg.), Gedächtnisschrift für Brigitte Knobbe-Keuk, 1997, S. 49 (97 ff.).

22 S. OLG Dresden JW 1917, 978; Riehm (Fn. 20), $\$ 275$ Rn. 59.1; G. Caspers, in: J. von Staudinger (Hrsg.), Kommentar zum Bürgerlichen Gesetzbuch, 2019, $\$ 275$ Rn. 23; T. Riehm, Der Grundsatz der Naturalerfüllung, 2015, S. 62 f.; G. Schiemann, in: J. von Staudinger (Hrsg.), Kommentar zum Bürgerlichen Gesetzbuch (Fn. 22), 2015, $\$ 243$ Rn. 20; E. Wolf, Anleitung zum Lösen zivilrechtlicher Fälle, JuS 1962, S. 101 (103 ff.). 
Auffassung muss (und darf) der Verkäufer daher seine Lieferpflicht erfüllen, wenn der aktuelle Vorrat für die Erfüllung des gerade geltend gemachten Anspruchs ausreicht; konkurrierende schuldrechtliche Ansprüche anderer Käufer bleiben insoweit außer Betracht. Erst wenn der Vorrat erschöpft ist, schuldet der Verkäufer auch keine weiteren Anstrengungen mehr, kann sich aber i.d.R. für die Nichtleistung exkulpieren, wenn und weil eine rechtfertigende Pflichtenkollision vorlag. ${ }^{23}$ Ebenso darf er selbst - wenn er zugleich Produzent ist - entscheiden, wie er seinen Betrieb organisiert, welche Bestellungen über welche Waren er bevorzugt behandelt, und welche er den beschränkten Kapazitäten opfert; auch hier wird man nicht verlangen können, dass er - gerichtlich überprüfbar! - seinen Betrieb so organisiert, dass alle Käufer verschiedenartiger Waren proportional befriedigt werden können.

\section{Grenzen der geschuldeten Anstrengungen}

Die vom Verkäufer geschuldeten Anstrengungen sind allerdings nicht unbegrenzt: Zunächst können die Parteien vertraglich von vornherein Beschränkungen der Leistungspflicht vorsehen. So kann etwa ein Händler als Verkäufer die geschuldete Gattung auf Waren aus dem eigenen Lager bzw. Vorrat beschränken und so die Verpflichtung zur Beschaffung weiterer Waren am freien Markt ausschließen. Gleiches gilt für die bereits erwähnte Beschränkung der geschuldeten Gattung auf die eigene Produktion beim Kauf vom Hersteller. Auch Begrenzungen des geschuldeten Beschaffungsaufwands bis hin zu einem freien Lösungsrecht des Verkäufers im Falle der Nichtbelieferung (Selbstbelieferungsvorbehalt) sind vertraglich vereinbar, sofern der Verkäufer einen kongruenten Deckungskauf abgeschlossen hatte, der dann scheiterte. ${ }^{24}$

a) Die wichtigste gesetzliche Grenze der geschuldeten Leistungsanstrengungen bildet zunächst $\$ 275$ Abs. 2 BGB. Diese Norm gewährt dem Verkäufer ein Leistungsverweigerungsrecht, wenn sein Aufwand im Verhältnis zum Leistungsinteresse des Gläubigers unverhältnismäßig groß ist. Die Einrede aus $\mathbb{2 7 5}$ Abs. 2 BGB besteht auch dann, wenn die Leistungser-

23 Vgl. T. Riehm, Pflichtverletzung und Vertretenmüssen, in: A. Heldrich/J. Prölss/I. Koller (Hrsg.), Festschrift für Claus-Wilhelm Canaris zum 70. Geburtstag, 2007, Bd. 1, S. 1079 (1101) sowie Riehm (Fn. 20), \$280 Rn. 175.

24 BGH NJW 1995, 1959; Riehm (Fn. 20), 275 Rn. 60; D. Joost, in: C. T. Ebenroth/K. Boujong/D. Joost/L. Strohn (Hrsg.), Handelsgesetzbuch, 3. Aufl. 2015, $\$ 346$ Rn. 119. 
schwerung nur vorübergehend auftritt - wovon bei der Covid-19-Pandemie auszugehen ist. Sie kann dann als dilatorische Einrede für die Dauer der Pandemie-bedingten Leistungserschwerungen erhoben werden. ${ }^{25} \mathrm{Al}-$ lerdings greift diese Grenze seltener, als auf den ersten Blick ersichtlich ist. Insbesondere greift sie bei Preissteigerungen auf dem vorgelagerten Markt nur selten. Der Vergleichsmaßstab für den Aufwand des Verkäufers als Schuldner ist nämlich nicht etwa der Kaufpreis, sondern das Leistungsinteresse des Gläubigers; die Gegenleistung wird in $\$ 275$ Abs. 2 BGB nicht erwähnt. Wenn nun Pandemie-bedingt bestimmte Güter am Markt teurer werden, steigt zwar der Leistungsaufwand für den Verkäufer, der die Waren zu höheren Preisen von seinen Lieferanten einkaufen muss. Wenn aber zugleich der Marktpreis auf der nächsten Handelsstufe steigt, erhöht sich das Leistungsinteresse des Käufers typischerweise gleichermaßen. Denn das Leistungsinteresse des Gläubigers entspricht in der Regel dem Schadensersatz statt der Leistung, den der Käufer verlangen könnte, ${ }^{26}$ und dieser wiederum umfasst mindestens die Kosten eines Deckungsgeschäfts, d.h. des Kaufes der geschuldeten Ware bei einem anderen Anbieter. Damit entspricht das Leistungsinteresse des Käufers (mindestens) dem gestiegenen Marktpreis auf der nächsten Handelsstufe. Solange Einkaufspreis des Verkäufers und Marktpreis auf der nächsten Handelsstufe parallel steigen, kann daraus also kein grobes Missverhältnis zwischen Leistungsaufwand des Verkäufers und Leistungsinteresse des Käufers folgen - selbst wenn der Leistungsaufwand auf ein Vielfaches des vereinbarten Kaufpreises steigen sollte. ${ }^{27}$

Dies zeigt sich am konkreten Beispiel von Webcams, deren Preise während der Covid-19-Pandemie erheblich gestiegen sind: Ein Verkäufer hatte eine Webcam für $100 €$ an einen Endkunden verkauft. Den Kaufpreis hatte er auf der Grundlage eines Einkaufspreises von $80 €$ kalkuliert. Wegen der stark gestiegenen Nachfrage erhöht sich sein Einkaufspreis nunmehr auf $160 €$. Der Marktpreis für dieselbe Kamera auf dem Endkundenmarkt ist inzwischen allerdings ebenfalls auf $200 €$ gestiegen. Da der Kunde die Kamera im Falle einer Nichtleistung des Verkäufers nunmehr anderweitig für $200 €$ kaufen müsste, beträgt sein Leistungsinteresse (mindestens) $200 €$. Der Leistungsaufwand des Verkäufers von $160 €$ ist im Hinblick hier-

25 Riehm (Fn. 20), \$275 Rn. 248 f.

26 BGH NJW 2008, 3122 (jedenfalls für zu vertretende Leistungshindernisse); Riehm (Fn. 20), \275 Rn. 219; W. Ernst, in: Münchener Kommentar zum Bürgerlichen Gesetzbuch, 8. Aufl. 2019, $\$ 275$ Rn. 97.

27 Lorenz (Fn. 12), $\$ 1$ Rn. 24; Riehm (Fn. 20), $\$ 275$ Rn. 220; Ernst (Fn. 26), $\$ 275$ Rn. 98. 
auf nicht grob unverhältnismäßig. Dass der Leistungsaufwand erheblich mehr als den vereinbarten Kaufpreis von $100 €$ beträgt, ist für die Anwendung des $\$ 275$ Abs. 2 BGB unerheblich. Es kann allenfalls ein Wegfall der Geschäftsgrundlage gem. $\$ 313$ Abs. 1 BGB vorliegen (Fallgruppe der Äquivalenzstörung), der zu einem Anspruch auf Vertragsanpassung und ggfs. zu einem Rücktrittsrecht der belasteten Partei (hier: des Verkäufers) führen kann. ${ }^{28}$

Fälle des $₫ 275$ Abs. 2 BGB werden daher allenfalls vorliegen, wenn Umstände spezifisch beim Verkäufer eintreten und dessen Kosten erhöhen, ohne dass zugleich der Marktpreis steigt. Das mag für einen Produzenten gelten, der durch höhere Hygieneanforderungen oder wegen Personalmangels seine Produktion drosseln muss, sodass seine Stückkosten steigen, während der Marktpreis für das Produkt konstant bleibt, weil Wettbewerber die gleiche Ware weiterhin zum bisherigen Preis anbieten. Andere denkbare Fälle sind solche, bei denen das Leistungsinteresse des Gläubigers infolge der Covid-19-Pandemie sogar sinkt, weil er selbst für die bestellte Ware keine Verwendung mehr hat. ${ }^{29}$ Hier ist eine Leistungsbefreiung aufgrund der Einrede aus $₫ 275$ Abs. 2 BGB denkbar, wenn der Aufwand des Produzenten in Relation zum Leistungsinteresse des Abnehmers - also faktisch zum Marktpreis oder einem ggfs. gesunkenen Interesse unverhältnismäßig groß wird. Wo diese Schwelle genau liegt, ist einzelfallabhängig und schwer allgemein zu bestimmen. ${ }^{30} \mathrm{Zu}$ berücksichtigen ist dabei insbesondere, ob der Käufer die Ware anderweitig am Markt erhalten kann, also nicht auf die Erfüllung durch diesen konkreten Verkäufer angewiesen ist. Für nicht zu vertretende Leistungserschwerungen, wie sie im Rahmen der Covid-19-Pandemie regelmäßig vorliegen dürften, wird man annehmen können, dass der Verkäufer - wenn die Ware anderweitig verfügbar ist - nur wenig mehr als den Marktpreis, den der Käufer bei Dritten bezahlen müsste, aufwenden muss (etwa 5-10\%). Ist der Käufer dagegen auf die Lieferung des Verkäufers angewiesen, wird man die Schwelle höher ansetzen müssen, z.B. bei einer Überschreitung des Leistungsinteresses um $10-20 \% .^{31}$

28 S. Weller/Lieberknecht/Habrich, Virulente Leistungsstörungen (Fn. 1), S. $1021 \mathrm{f}$. m.w.N. sowie Prütting, in diesem Band.

29 Weller/Lieberknecht/Habrich, Virulente Leistungsstörungen (Fn. 1), S. 1020.

30 Für Anhaltspunkte s. Riehm (Fn. 20), $\$ 275$ Rn. 213 ff.; für konkrete Zahlen I. Bach, Leistungshindernisse, 2017, S. $428 \mathrm{ff}$.

31 Ähnlich Ernst (Fn. 26), $\$ 275$ Rn. 107 ff. (ohne konkrete Zahlen); abweichende Zahlenverhältnisse (allein in Abhängigkeit vom Verschuldensgrad) bei F. Faust, in: P. Huber/F. Faust (Hrsg.), Schuldrechtsmodernisierung, 2002, Kap. 2 Rn. 68. 
b) Eine weitere Grenze für die geschuldeten Leistungsanstrengungen des Schuldners bildet die „echte“ Unmöglichkeit gem. $\$ 275$ Abs. 1 BGB. Die Leistung ist unmöglich im Sinne dieser Vorschrift, wenn naturgesetzlich ausgeschlossen oder rechtlich verboten ist, dass der Verkäufer liefert. Das kann beispielsweise der Fall sein, wenn der Markt für den Verkäufer vollständig „leergefegt“ ist, sodass unabhängig von seinen Anstrengungen keine Ware mehr zu erhalten ist, etwa weil sämtliche Produktionsstätten geschlossen sind. Die tatsächliche Unmöglichkeit wird allerdings nur vorübergehend sein, weil davon auszugehen ist, dass die aktuellen Regelungen, Betriebsschließungen etc. eines Tages wieder aufgehoben werden, sodass die Leistungshindernisse entfallen werden. ${ }^{32}$ Gleichwohl sind dann für die Zeit des Hindernisses keine Anstrengungen geschuldet, weil sie ohnehin sinnlos wären. Gleiches gilt für vorübergehende rechtliche Leistungshindernisse, wenn also die geschuldete Leistung verboten ist. Das wird bei gewöhnlichen Kaufverträgen im Handel auch in Corona-Zeiten kaum jemals der Fall sein, solange nicht die Lieferung bestimmter Gegenstände (z.B. Schutzmasken) behördlich verboten wird - anders als bei Dienst- und Werkleistungen. Denkbar ist dies aber bei Kaufverträgen mit Herstellern, deren Betrieb durch behördliche Anordnung geschlossen wurde, sodass sie die geschuldeten Waren ohne Rechtsverstoß nicht herstellen können. Hier liegt tatsächlich ein Fall (vorübergehender) rechtlicher Unmöglichkeit vor, in welchem auch keine Leistungsanstrengungen geschuldet sind.

c) Die letzte Grenze der geschuldeten Leistungsanstrengungen bildet ein möglicher Wegfall der Geschäftsgrundlage. Die Leistungspllicht findet dort ihre Grenze, wo der Vertrag nach $₫ 313$ Abs. 1 BGB angepasst werden muss, weil die Veränderung der Umstände so gravierend ist, dass ein Festhalten am unveränderten Vertrag einer Partei unter Berücksichtigung der vertraglichen und gesetzlichen Risikoverteilung nicht mehr zugemutet werden kann. Das kann der Fall sein, wenn die Aufwendungen des Verkäufers den vereinbarten Kaufpreis infolge der Covid-19-Pandemie deutlich übersteigen. Diese Frage ist allerdings nicht Gegenstand dieses Beitrags. ${ }^{33}$

d) Keine relevante Grenze für den kaufrechtlichen Lieferanspruch bildet dagegen die persönliche Unzumutbarkeit gemäß $\ 275$ Abs. 3 BGB: Diese Vorschrift ist nur auf höchstpersönliche Leistungspflichten anwendbar, de-

32 Weller/Lieberknecht/Habrich, Virulente Leistungsstörungen (Fn. 1), S. 1020.

33 S. dazu Prütting, in diesem Band. 
ren Erfüllung auch nicht auf Dritte delegiert werden darf. ${ }^{34}$ Die kaufvertragliche Lieferpflicht ist in aller Regel keine solche, weil es dem Käufer nur auf die Ware ankommt, nicht dagegen auf die Person des Verkäufers. Selbst wenn daher einem konkreten Verkäufer die Erfüllung aus persönlichen Gründen unzumutbar sein sollte, etwa weil er wegen seiner Zugehörigkeit zu einer Risikogruppe jedes Infektionsrisiko vermeiden und daher im Rahmen der Lieferung nicht in Kontakt zum Kunden treten möchte, spricht in diesem Fall nichts gegen eine Delegation der Lieferpflicht auf Dritte - selbst wenn diese mit Mehrkosten verbunden sein sollte. Die Frage, inwieweit diese Mehrkosten aufzubringen sind, beantwortet aus-

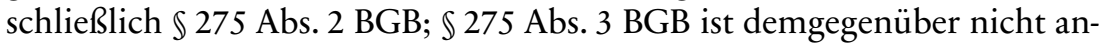
wendbar. ${ }^{35}$

e) Die vorstehend dargestellten Maßstäbe beschreiben nicht nur die Grenzen des Erfüllungsanspruches, sondern zugleich den Umfang der „im Verkehr erforderlichen Sorgfalt" im Sinne von $\$ 276$ Abs. 2 BGB. Daher hat ein Verkäufer seine Nichtleistung zu vertreten i.S.v. $\$ 280$ Abs. 1 S. 2 bzw. $\$ 286$ Abs. 4 BGB, wenn er diesen Maßstab unterschreitet. Hat der Verkäufer darüber hinaus sogar eine Garantie für die Belieferung übernommen, also vertraglich erklärt, dass er für die Folgen einer Nichtleistung unabhängig von seinem Verschulden einstehen werde, so kommt es auf ein Verschulden im vorstehend beschriebenen Sinne gar nicht an: Er hat seine Nichtleistung immer dann zu vertreten, wenn das betroffene Risiko von seiner Garantie umfasst ist. Daher ist insoweit zunächst der genaue Wortlaut der Garantieerklärung zu untersuchen, ob er auch ein Pandemierisiko umfasst. ${ }^{36}$ Finden sich im Wortlaut keine Anhaltspunkte hierfür, ist im Wege der Auslegung weiter zu prüfen, ob dies zumindest dem Willen der Parteien entsprach. Im Hinblick darauf, dass die Covid-19-Pandemie ein völlig unvorhersehbares Risiko darstellt, wird man nicht ohne weiteres annehmen können, dass eine Liefergarantie auch dieses umfasst.

34 Vgl. M. Weller, Persönliche Leistungen, 2012, S. $21 \mathrm{ff} ., 241 \mathrm{ff} . ;$ s. auch Ernst (Fn. 26), \275 Rn. 117; C.-W. Canaris, Das Leistungsverweigerungsrecht wegen Unzumutbarkeit der Leistung in einer Kollisions- oder Konfliktslage nach deutschem Recht, in: M. V. de Giorgi/S. Delle Monache/G. de Cristofaro (Hrsg.), Studi in onore di Giorgio Cian, 2010, S. 383 (385, 388 f.).

35 Näher Riehm (Fn. 20), \$275 Rn. 265 ff.

36 S. auch unten D.IV zu den Folgen der Übernahme eines Beschaffungsrisikos. 


\section{Allgemeine Rechtsfolgen}

Unabhängig davon, ob der Verkäufer die erforderlichen Lieferanstrengungen unterschreitet oder nicht, ob er also die Nichtleistung zu vertreten hat oder nicht, kann der Käufer im Falle einer Nichterfüllung durch den Verkäufer zunächst nach $\$ 320$ BGB den Kaufpreis zurückhalten, sofern dieser noch nicht bezahlt ist. Darüber hinaus kann er nach $\$ 323$ Abs. 1 BGB dem Verkäufer eine Frist zur Lieferung setzen und nach deren fruchtlosem Ablauf vom Vertrag zurücktreten. Der Verkäufer schuldet dann die Erstattung eines evtl. bereits bezahlten Kaufpreises ( $\$ 346$ Abs. 1 BGB).

\section{Rechtsfolgen bei zu vertretender Nichtleistung}

Nunmehr sind die Rechtsfolgen zu untersuchen, die eintreten, wenn die Leistungsanstrengungen des Verkäufers hinter dem geschuldeten Maß zurückbleiben und er deswegen nicht leistet. Derartige Fälle dürften nicht selten sein, etwa wenn ein Verkäufer nicht bereit ist, Aufwendungen zu erbringen, die den Kaufpreis überschreiten, ohne dass ein Fall des $₫ 313$ Abs. 1 BGB bzw. eines vertraglichen Selbstbelieferungsvorbehalts vorliegt.

In diesem Fall kommt der Verkäufer nach einer Mahnung des Käufers in Lieferverzug. Dementsprechend schuldet er dem Käufer den Ersatz des Verzögerungsschadens, etwa Anwaltskosten zur Durchsetzung seines Anspruchs ( $\mathbb{S} 280$ Abs. 1, Abs. 2, 286 BGB). Hinzu kommt, dass der Käufer dem Verkäufer eine Nachfrist zur Lieferung setzen kann und nach deren fruchtlosem Ablauf berechtigt ist, Schadensersatz statt der Leistung zu verlangen ( $\$ \$ 280$ Abs. 1, Abs. 3, 281 Abs. 1 BGB). Das bedeutet im praktischen Ergebnis, dass er sich die ausgebliebene Leistung nunmehr am freien Markt beschaffen kann (soweit möglich), und etwaige Mehrkosten vom Verkäufer ersetzt erhält. Voraussetzung ist lediglich, dass der Verkäufer mit seinen Anstrengungen zur Lieferung hinter denjenigen zurückbleibt, die er nach den vorstehenden Ausführungen schuldet ( $\$ 280$ Abs. 1 S. 2 BGB).

Am obigen Beispiel der Webcam illustriert bedeutet dies, dass der Käufer nunmehr, wenn der Verkäufer die für $100 €$ verkaufte Kamera trotz Nachfristsetzung nicht liefert, die Kamera für $200 €$ bei der Konkurrenz kaufen und die Differenz von $100 €$ vom Verkäufer verlangen könnte. Der Verkäufer, der nicht bereit war, die Kamera für $160 €$ am Markt zu kaufen und damit $60 €$ Verlust gegenüber dem Vertragspreis zu machen, verlöre dadurch selbst den Vertragspreis und müsste zusätzliche $100 €$ an den Käufer bezahlen. Auch dies gilt selbstverständlich nur unter der Vorausset- 
zung, dass keine Vertragsanpassung nach $₫ 313$ Abs. 1 BGB stattfindet. Freilich könnte gerade dieses Beispiel ein typischer Fall einer Äquivalenzstörung sein, die von $\$ 313$ BGB erfasst wird. ${ }^{37}$

\section{Rechtsfolgen bei nicht zu vertretender Nichtleistung}

Abschließend seien noch die Rechtsfolgen untersucht, die eintreten, wenn die Anstrengungen, die der Verkäufer unternehmen müsste, um die Leistung noch $\mathrm{zu}$ erbringen, das nach dem Vorstehenden geschuldete Maß überschreiten. In diesem Fall ist die Leistung entweder vorübergehend tatsächlich oder rechtlich unmöglich, beispielsweise weil der Verkäufer wegen einer Betriebsschließung oder gar der Beschlagnahmung der Ware (man denke etwa an Schutzmasken) seine Vertragsplicht nicht erfüllen kann. Oder die Erfüllung erfordert vorübergehend Aufwendungen, die grob außer Verhältnis zum Leistungsinteresse des Käufers stehen.

Im ersten Fall ist der Erfüllungsanspruch analog $\mathbb{} 275$ Abs. 1 BGB vorübergehend suspendiert, solange das Hindernis besteht; im zweiten Fall kann der Verkäufer analog 275 Abs. 2 BGB vorübergehend die Einrede des grob unverhältnismäßigen Leistungsaufwandes gegen die Inanspruchnahme durch den Käufer erheben. Da die Leistungshindernisse nicht endgültig sind, liegt allerdings keine echte Unmöglichkeit im Sinne von $\$ 275$ Abs. 1 BGB vor, und auch die Einrede der groben Unverhältnismäßigkeit ist in diesem Fall keine dauernde, sondern nur eine vorübergehende. Vielmehr wäre eine Klage des Käufers auf Lieferung nach der zutreffenden h.M. nur als „derzeit unbegründet“ abzuweisen. ${ }^{38}$ Möglich ist aber gleichwohl eine Klage auf künftige Leistung unter den Voraussetzungen des $\$ 259 \mathrm{ZPO} .{ }^{39}$

37 Vgl. Prütting, in diesem Band; Weller/Lieberknecht/Habrich, Virulente Leistungsstörungen (Fn. 1), S. $1021 \mathrm{f}$.

38 Riehm (Fn. 20), \$275 Rn. 154 ff. m.w.N.

39 Vgl. Lorenz (Fn. 11), $\$ 275$ Rn. 73 (für den Fall des Ausstehens einer behördlichen Genehmigung); Ernst (Fn. 26), $\$ 275$ Rn. 141; Bach, Leistungshindernisse (Fn. 30), S.777; C.-W. Canaris, Die einstweilige Unmöglichkeit der Leistung, in: T. Baums/M. Lutter/K. Schmidt/J. Wertenbruch (Hrsg.), Festschrift für Ulrich Huber zum siebzigsten Geburtstag, 2006, S. 143 (146 f.); W. Däubler, Die vorübergehende Unmöglichkeit der Leistung, in: S. Lorenz/A. Trunk/H. Eidenmüller/C. Wendehorst/J. Adolff (Hrsg.), Festschrift für Andreas Heldrich zum 70. Geburtstag, 2005, S. 55 (59); B. Gsell, Rechtskräftiges Leistungsurteil und Klage auf Schadensersatz statt der Leistung, JZ 2004, S. 110 (114); D. Kaiser, Zeitweilige Unmöglichkeit, in: F. Häuser/H. Hammen/J. Hennrichs/A. Steinbeck/U. R. Siebel/R. 
Da keine echten Fälle des $₫ 275$ BGB vorliegen, sind auch die Vorschriften der $\$ \$ 283,311$ a, 326 BGB nicht anwendbar. Weder fällt daher der Erfüllungsanspruch weg und wird durch einen Schadensersatzanspruch ersetzt, noch entfällt automatisch der Anspruch auf die Gegenleistung; ${ }^{40}$ vielmehr sind die Sekundärfolgen vorübergehender Leistungshindernisse nicht gesetzlich geregelt. Nach zutreffender h.M. sind insofern die Vorschriften über Leistungsverzögerungen entsprechend anzuwenden, wobei auf das Kriterium der „Durchsetzbarkeit“ der Forderung als Voraussetzung für Sekundärrechte verzichtet wird, weil diese während des Bestehens des Hindernisses gerade fehlt. ${ }^{41}$ Dabei ist in aller Regel davon auszugehen, dass der Verkäufer die Leistungsverzögerung nicht zu vertreten hat i.S.v. $\$ 276$ Abs. 1 BGB. Denn die Covid-19-Pandemie ist kein Umstand, den er bei Anwendung der verkehrserforderlichen Sorgfalt hätte vorhersehen und vermeiden können; das gilt in der Regel selbst dann, wenn der Vertrag zu einem Zeitpunkt abgeschlossen wurde, zu welchem die Auswirkungen der Pandemie bereits vorhersehbar waren, sofern und weil sie auch dann für den Verkäufer jedenfalls nicht vermeidbar waren. Und selbst wenn der Verkäufer ein Beschaffungsrisiko gem. $\$ 276$ Abs. 1 S. 1 a.E. BGB übernommen hat, wie das bei der Vereinbarung einer Gattungsschuld regelmäßig der Fall ist, umfasst dieses in aller Regel nur typische Beschaffungsprobleme wie etwa (übliche) Preissteigerungen auf dem vorgelagerten Markt und die Erschließung von Bezugsquellen, nicht aber spezifisch pandemiebedingte Lieferschwierigkeiten. ${ }^{42}$

Dementsprechend kommt der Verkäufer in diesen Fällen nicht in Lieferverzug ( $\$ 286$ Abs. 4 BGB), schuldet also keinen Ersatz der Verzögerungsschäden des Käufers. Zudem besteht keine Grundlage für einen Anspruch des Käufers auf Schadensersatz statt der Leistung gem. $\mathbb{S} 280$ Abs. 1, Abs. 3, 281 BGB, sodass er auch etwaige Mehrkosten, die durch ein Deckungsgeschäft am Markt entstehen, nicht vom Verkäufer ersetzt verlangen kann.

Unberührt bleiben allerdings die verschuldensunabhängigen Rechtsbehelfe. Danach kann der Käufer gemäß $₫ 320$ BGB selbstverständlich die

Welter (Hrsg.), Festschrift für Walther Hadding zum 70. Geburtstag am 8. Mai 2004, 2004, S. 121 (129); vgl. auch RG JW 1919, 188 f.; a.A. F. Eichel, Künftige Forderungen, 2014, S. 323.

40 Riehm (Fn. 20), \$275 Rn. 159.

41 Riehm (Fn. 20), $\$ 275$ Rn. 161 m.w.N. auch zu abweichenden dogmatischen Erklärungen.

42 R. Schaub, in: beck-online.Großkommentar zum Zivilrecht (Fn. 14), 1.3.2020, \$276 Rn. 156; Bacher, Corona-Pandemie (Fn. 7), S. 5168 f. 
Zahlung des Kaufpreises verweigern, solange der Verkäufer zur Lieferung der Ware nicht in der Lage ist. ${ }^{43}$ Das gilt gemäß $\$ 321$ BGB auch dann, wenn der Käufer zur Vorleistung verpflichtet ist. ${ }^{44} \mathrm{Ob}$ er einen bereits bezahlten Kaufpreis für die Zwischenzeit zurückverlangen kann, bis der Verkäufer wieder leistungsfähig ist, ist umstritten: Ein Teil der Literatur bejaht einen solchen vorläufigen Rückforderungsanspruch analog $\$ 326$ Abs. 4 BGB. ${ }^{45}$ Nach hier vertretener Auffassung ist ein solcher Anspruch dagegen abzulehnen, weil dem Käufer ohnehin ein Rücktrittsrecht nach $\$ 323$ BGB zusteht, aufgrund dessen er einen bereits bezahlten Kaufpreis zurückverlangen kann - dann allerdings unter vollständiger Vertragsaufhebung. Möchte er jedoch am Kaufvertrag festhalten und die Lieferung zu einem späteren Zeitpunkt weiterhin beanspruchen, so besteht kein Grund, von einer vertraglich vereinbarten Vorleistungspflicht mit entsprechenden Fälligkeitsterminen abzuweichen. Selbst wenn dadurch die Vorleistung dem Insolvenzrisiko des Verkäufers ausgesetzt wird, realisiert sich damit nur dasjenige Risiko, das mit einer ungesicherten Vorleistung durch den Käufer stets verbunden ist. ${ }^{46}$ Abweichungen hiervon sind nach hier vertretener Auffassung nur aufgrund des $₫ 313$ BGB zuzulassen.

Unbenommen bleibt dem Käufer allerdings nach h.M. das Rücktrittsrecht nach $\$ 323$ BGB. ${ }^{47}$ Dieses hängt nach dem eindeutigen Gesetzeswortlaut nicht davon ab, dass der Verkäufer die Nichtleistung zu vertreten hat. Zwar setzt dieses Rücktrittsrecht nach zutreffender h.M. voraus, dass eine „fällige und durchsetzbare“ Leistungspflicht besteht, woran man im Falle der vorübergehenden Unmöglichkeit bzw. der vorübergehenden Einrede

43 S. auch Bacher, Corona-Pandemie (Fn. 7), S. 518.

44 S. zum daraus folgenden Rücktrittsrecht gem. $\int \mathbb{S} 321$ Abs. 2, 323 BGB A. Arnold, Die vorübergehende Unmöglichkeit nach der Schuldrechtsreform, JZ 2002, S. 866 (869).

45 Bach, Leistungshindernisse (Fn. 30), S. 779 f.; H. P. Westermann, in: W. Erman (Hrsg.), Bürgerliches Gesetzbuch, 15. Aufl. 2017, 275 Rn. 12; Canaris, Einstweilige Unmöglichkeit (Fn. 39), S. 150; Faust (Fn. 31), Kap. 8 Rn. 8; E. Wieser, Schuldrechtsreform - Die Unmöglichkeit der Leistung nach dem neuen Recht, MDR 2002, S. 858 (861); C.-W. Canaris, Die Reform des Rechts der Leistungsstörungen, JZ 2001, S. 499 (500); krit. Arnold, Vorübergehende Unmöglichkeit (Fn.44), S. 868.

46 Riehm (Fn. 20), 275 Rn. 167; Ernst (Fn. 26), Rn. 144; P. Schmidt, Die Unmöglichkeit der Erfüllung in Ansehung der Zeit, 2007, S. $99 \mathrm{f}$.

47 Ebenso Caspers (Fn. 22), \275 Rn. 50; Ernst (Fn. 26), $\$ 275$ Rn. 151 f.; Canaris, Einstweilige Unmöglichkeit (Fn. 39), S. 151 ff.; Kaiser, Zeitweilige Unmöglichkeit (Fn. 39), S. 134 f.; T. Lobinger, Die Grenzen rechtsgeschäftlicher Leistungspflichten, 2004, S. $316 \mathrm{ff}$. 
nach $\$ 275$ Abs. 2 BGB zweifeln könnte; ${ }^{48}$ der Zweck der "Suspendierung“ der Lieferpflicht wegen des vorübergehenden Hindernisses steht einer entsprechenden Anwendung des $₫ 323$ BGB jedoch nicht entgegen. ${ }^{49}$ Dementsprechend kann der Käufer dem Verkäufer eine angemessene Nachfrist zur Lieferung setzen und nach deren fruchtlosem Ablauf vom Vertrag zurücktreten. Bei der Bemessung der Angemessenheit der Frist ist, solange ein Ende des Leistungshindernisses nicht absehbar ist, vorrangig auf das Verwendungsinteresse des Käufers abzustellen, d.h. darauf, wie dringend er die Ware benötigt. Nach $\$ 323$ Abs. 4 BGB besteht das Rücktrittsrecht sogar schon vor Fälligkeit, wenn absehbar ist, dass die pandemiebedingten Leistungshindernisse auch am Fälligkeitstag noch bestehen werden.

In der Literatur wird zudem befürwortet, die vorübergehende Unmöglichkeit bzw. die vorübergehenden Leistungserschwerungen dann der „echten“ (dauernden) Unmöglichkeit gem. $\$ 242$ BGB gleichzustellen, wenn das weitere Zuwarten dem Käufer nicht mehr zumutbar sei, und wenn auch dem Schuldner nicht mehr zugemutet werden könne, den Vertrag nach Beseitigung des Hindernisses noch zu erfüllen. ${ }^{50}$ Danach würden sich die Rechtsfolgen aus den $\mathbb{S} 283,326$ ergeben, sobald die Unmöglichkeit gem. $\$ 242 \mathrm{BGB}$ als dauernde zu behandeln wäre. Allerdings führen diese Vorschriften zu einem automatischen Übergang vom Erfüllungsanspruch auf die Sekundärrechte, ohne dass es einer entsprechenden Gestaltungserklärung des Gläubigers bedürfte. Das führt zu erheblicher Rechtsunsicherheit, weil erst ex post vom Gericht feststellbar ist, ob die Voraussetzungen für diese einschneidenden Rechtsfolgen vorlagen. Zudem bewirkt sie eine Bevormundung des Gläubigers, weil dieser nicht mehr selbst darüber entscheiden kann, ob er noch an den Vertrag gebunden sein will oder nicht. Vorzugswürdig ist demgegenüber eine Anwendung der Grundsätze des $₫ 323$ Abs. 2 Nr. 3 BGB,${ }^{51}$ wonach dem Käufer ein Rücktrittsrecht ohne vorhergehende Fristsetzung zusteht, wenn ihm das Zuwarten bis

48 So etwa Kaiser, Zeitweilige Unmöglichkeit (Fn. 39), S. $136 \mathrm{f}$.

49 Näher Riehm (Fn. 20), \$275 Rn. 165, 161 f.

50 So Bacher, Corona-Pandemie (Fn. 7), S. 517; Lorenz (Fn. 12), $\mathbb{1}$ Rn. 14; Caspers (Fn. 22), $\$ 275$ Rn. 53 ff.; R. Schwarze, Das Recht der Leistungsstörungen, 2. Aufl. 2017, \4 Rn. 24; Westermann (Fn. 45), \$275 Rn. 12; B. Gsell, in: T. Soergel (Hrsg.), Bürgerliches Gesetzbuch mit Einführungsgesetz und Nebengesetzen, 13. Aufl. 2013, $\$ 311$ a Rn.30; D. Medicus, Bemerkungen zur „vorübergehenden Unmöglichkeit“, in: S. Lorenz et al. (Hrsg.), FS Heldrich (Fn. 39), S. 347 (351, 354 ff.); Faust (Fn. 31), Kap. 8 Rn. 9.

51 Die Beschränkung des $₫ 323$ Abs. 2 Nr. 3 auf Schlechtleistungen hat insoweit - auBerhalb des Anwendungsbereichs der Verbraucherrechterichtlinie - keine praktischen Auswirkungen, vgl. D. Looschelders, in: beck-online.Großkommentar zum 
zum Ablauf einer angemessenen Nachfrist unter Abwägung der beiderseitigen Interessen nicht mehr zumutbar ist. ${ }^{52}$ Das Erfordernis einer Rücktrittserklärung in diesen Fällen schafft nach hier vertretener Auffassung mehr Rechtssicherheit als der von der h.M. angenommene automatische Übergang zu den Sekundärrechten nach $\$ 242$ BGB.

Die Nachfristsetzung ist zudem gemäß $₫ 323$ Abs. 2 Nr. 2 BGB bzw. $\$ 376$ HGB entbehrlich, wenn ein sogenanntes „relatives Fixgeschäft" vorliegt, der Käufer im Vertrag also sein Leistungsinteresse an die Einhaltung der vereinbarten Leistungszeit gebunden hat. ${ }^{53}$ Auch hierfür kommt es nicht darauf an, ob die Überschreitung der Leistungszeit vom Verkäufer zu vertreten ist.

Im praktischen Ergebnis wird dadurch der Vertrag während des Bestehens der pandemiebedingten Leistungshindernisse grundsätzlich beidseitig suspendiert; nur wenn auf Seiten des Käufers eine besondere Dringlichkeit gegeben ist, kann dieser ein Rücktrittsrecht entweder nach Nachfristsetzung oder nach dem Gedanken des $\$ 323$ Abs. 2 Nr. 3 BGB ausüben. Diese Lösung erscheint beidseits interessengerecht, sodass im Regelfall kein Bedarf nach einer Vertragsanpassung oder gar einem Rücktrittsrecht nach $₫ 313$ BGB bestehen dürfte.

\section{Störungen auf Seiten des Käufers}

Denkbar ist schließlich auch, dass der Verkäufer zwar zur Lieferung in der Lage ist, der Käufer die Waren allerdings pandemiebedingt nicht abnimmt. Hierzu ist zunächst festzustellen, dass der bloße Umstand, dass der Käufer infolge der Covid-19-Pandemie keinen Bedarf mehr für die gekauften Waren hat oder diese nicht mehr bezahlen kann, diesen nicht von seiner Abnahmepflicht nach $₫ 433$ Abs. 2 BGB befreit. Beide Umstände fallen in seinen Risikobereich und berühren weder die Wirksamkeit des Vertrages noch die Durchsetzbarkeit der daraus folgenden Pflichten. Abhilfe kann insoweit allenfalls ein Wegfall der Geschäftsgrundlage gemäß $₫ 313$

Zivilrecht (Fn. 14), 01.03.2020, \$323 Rn. 202 ff.; T. Riehm, Irrungen und Wirrungen zur Fristsetzung und ihrer Entbehrlichkeit, NJW 2014, S. 2065 (2068).

52 S. näher Riehm (Fn. 20), $\$ 275$ Rn. $174 \mathrm{ff}$.

53 Eingehend dazu R. Schwarze, „Steht und fällt" - Das Rätsel der relativen Fixschuld, AcP 207 (2007), S. 437 ff. 
BGB schaffen, wobei allerdings zu berücksichtigen ist, dass das Verwendungsrisiko grundsätzlich gesetzlich dem Käufer zugewiesen ist. ${ }^{54}$

Ist der Käufer wegen der Auswirkungen der Covid-19-Pandemie faktisch nicht mehr in der Lage, die Ware anzunehmen, etwa weil er seinen Betrieb schließen musste, so ändert auch das nichts daran, dass er in Annahmeverzug gemäß $\$ \$ 293 \mathrm{ff}$. BGB gerät. Danach schuldet er etwa verschuldensunabhängig Ersatz der Mehraufwendungen des Verkäufers, z.B. für die zwischenzeitliche Einlagerung und eine erneute Anlieferung ( $\$ 304$ BGB). Dagegen hat die hierin zugleich liegende Verletzung der echten Pflicht zur Abnahme ( $\$ 433$ Abs. 2 Alt. 2 BGB) keine Konsequenzen, weil diese nicht zu vertreten ist bzw. der entsprechende Anspruch des Verkäufers auf Abnahme nach $₫ 275$ BGB ausgeschlossen ist.

\section{E. Leistungsstörungen beim Werkvertrag}

Beim Werkvertrag können pandemiebedingte Störungen sowohl auf Seiten des Unternehmers als auch auf Seiten des Bestellers auftreten.

\section{Störungen auf Seiten des Unternehmers}

Auf Seiten des Unternehmers geht es vor allem um Personalengpässe einerseits und um Materialengpässe andererseits. Z.B. kann ein Bauunternehmer den Vertrag nicht erfüllen, weil er wegen Krankheit, Quarantäne oder Betreuungsschwierigkeiten nicht genug Personal hat, das auf die Baustelle kommen kann, oder weil erforderliches Material pandemiebedingt nicht verfügbar ist - z.B. weil Baumärkte auch für professionelle Abnehmer geschlossen sind. Dabei sind auch bei Werkverträgen, insbesondere im Bereich des komplexen Anlagenbaus, Force Majeure-Klauseln verbreitet; insoweit gilt zunächst das oben zu B.I Ausgeführte. ${ }^{55}$

54 Statt aller S. Martens, in: beck-online.Großkommentar zum Zivilrecht (Fn. 14), 1.4.2020, $₫ 313$ Rn. 69 f.

55 S. dazu auch L. Weiser, Das Coronavirus und seine Auswirkungen auf den Bauablauf, NZBau 2020, S. 203 (204). 


\section{Bauverträge nach der $V O B / B$}

Im Hinblick auf Bauverträge sind ferner die Regeln der VOB/B zu beachten, falls diese vereinbart wurden. Dort enthält $\$ 6$ VOB/B Sonderregelungen für die Verzögerung des Bauablaufs infolge höherer Gewalt oder durch Umstände, die dem Risikobereich des Bestellers zuzuordnen sind. Diese Vorschrift führt ebenfalls zu einer Art „Suspendierungslösung“, indem die vereinbarten Ausführungsfristen während der Dauer der Verzögerung infolge höherer Gewalt verlängert werden. Es ist insoweit lediglich eine Behinderungsanzeige des Unternehmers erforderlich, d.h. der Bauunternehmer muss dem Bauherrn mitteilen, dass er pandemiebedingt nicht in der Lage ist, den Bau fortzusetzen. Während dieser Zeit kommt der Unternehmer nicht in Verzug; es fallen keine Vertragsstrafen an ( $\$ 339$ S. 1 BGB), und der Unternehmer schuldet mangels Fälligkeit keinen Schadensersatz wegen Verzögerung seiner Leistung. Mangels Fertigstellung und Abnahme wird allerdings auch der Werklohnanspruch nicht fällig. In der Gesamtschau bleibt der Vertrag dadurch gewissermaßen in der Schwebe, ohne dass weitere Konsequenzen eintreten. Allerdings können nach $\$ 6$ Abs. 7 VOB/B nach 3 Monaten beide Seiten den Bauvertrag kündigen, wenn sie nicht mehr länger zuwarten wollen. In diesem Fall wird dann eine Teilvergütung fällig, die sowohl die bereits erbrachten Leistungen umfasst als auch dasjenige, was der Bauunternehmer während der Verzögerung zusätzlich aufwenden musste, um sich leistungsbereit zu halten.

Inwieweit die Covid-19-Pandemie einen Fall höherer Gewalt im Sinne dieser Regelung darstellt bzw. in die Risikosphäre des Unternehmers fällt, hängt im Einzelfall davon ab, worin die Störung konkret besteht. ${ }^{56}$ Höhere Gewalt im Baurecht wird verstanden als „ein betriebsfremdes, von außen durch elementare Naturkräfte oder durch Handlungen dritter Personen herbeigeführtes Ereignis, das nach menschlicher Einsicht und Erfahrung unvorhersehbar ist, mit wirtschaftlich erträglichen Mitteln auch durch die äußerste nach der Sachlage vernünftigerweise zu erwartende Sorgfalt nicht verhütet oder unschädlich gemacht werden kann und auch nicht wegen seiner Häufigkeit vom Betriebsunternehmen in Kauf zu nehmen ist. " ${ }^{\text {" } 7}$ Das wird man im Falle pandemiebedingter Einschränkungen, die den Unternehmer treffen, grundsätzlich bejahen können, weil von einem Bauunternehmer vernünftigerweise nicht erwartet werden kann, dass er Dispositionen triff (etwa zusätzliches Personal vorhält oder Material lagert), um 
selbst die - noch nie dagewesenen - Folgen der Covid-19-Pandemie und der daraus folgenden behördlichen Maßnahmen abfedern zu können. Etwas anderes wird man allenfalls dann annehmen können, wenn der Bauvertrag nach Bekanntwerden des Überspringens der Pandemie nach Deutschland abgeschlossen wurde, sodass ein äußerst sorgfältiger Bauunternehmer mit entsprechenden Einschränkungen rechnen konnte. In allen anderen Fällen pandemiebedingter Störungen auf Seiten des Unternehmers dürfte es sich um höhere Gewalt im Sinne von $\$ 6$ Abs. 2 Nr. 1 lit. c VOB/B handeln..$^{58}$ Ist die Störung dem Risikobereich des Bestellers zuzuordnen (etwa eine Sperrung der Baustelle durch die Gesundheitsbehörde), so greift die Regelung gem. $\$ 6$ Abs. 2 Nr. 1 lit. b VOB/B ohnehin ein.

\section{Sonstige Werkverträge}

Auf reine BGB-Werkverträge ist $\$ 6 \mathrm{VOB} / \mathrm{B}$ nicht anzuwenden. Vielmehr ist nach den Regeln des Allgemeinen Leistungsstörungsrechts zu differenzieren: Pandemiebedingte Leistungsverzögerungen, die der Unternehmer bei Anwendung der im Verkehr erforderlichen Sorgfalt nicht durch vorsorgliche Dispositionen hätte abwenden können, hat dieser nicht zu vertreten. Gemäß $\$ 286$ Abs. 4 BGB kommt er daher nicht in Leistungsverzug, sodass ebenfalls keine Vertragsstrafen fällig werden ( $\$ 339$ S. 2 BGB) und kein Schadensersatz wegen Verzögerung der Leistung geschuldet ist. Aus dem gleichen Grund scheidet ein Anspruch des Bestellers auf Schadensersatz statt der Leistung nach $\$ 281$ BGB nach einer fruchtlosen Fristsetzung aus ( $\$ 280$ Abs. 1 S. 2 BGB). Typischerweise liegt aber ein Fall vorübergehender Unmöglichkeit vor, sodass der Erfüllungsanspruch des Bestellers für die Dauer des Leistungshindernisses (z.B. des Betretungsverbots auf der Baustelle, des Veranstaltungsverbots für Konzertveranstaltungen oder der Covid-19-Erkrankung des Solokünstlers bei höchstpersönlichen Leistungspflichten ${ }^{59}$ ) analog $\$ 275$ Abs. 1 BGB suspendiert ist.

58 Ebenso H. Fuchs/M. Dreher, Bau- und vergaberechtliche Herausforderungen durch die Corona-Pandemie, NZBau 2020, S. 201 (201); Weiser, Coronavirus (Fn. 55), S. 205.

59 K. Spenner/B. Estner, Absage von Veranstaltungen wegen des Coronavirus - wer zahlt?, BB 2020, S. 852 (853). 
a) Kündigungsrecht des Bestellers (\$S $648 \mathrm{f}$. BGB)

Selbstverständlich kann der Besteller jederzeit den Werkvertrag nach $₫ 648$ BGB kündigen; dieses Kündigungsrecht ist für ihn allerdings unattraktiv, weil er dann den gesamten Werklohn abzüglich der ersparten Aufwendungen des Unternehmers schuldet. Ein Kündigungsrecht des Bestellers aus wichtigem Grund gemäß $₫ 648$ a BGB wird man jedenfalls bei Bauverträgen allenfalls in extremen Ausnahmefällen annehmen können, weil ihm in der Regel trotz der vorübergehenden Störung zugemutet werden kann, den Vertrag nach Ende der Störung fortzusetzen. Anerkanntermaßen besteht ein Kündigungsrecht nach $\$ 648$ a BGB allerdings dann, wenn der Unternehmer sich weigert, der gebotenen Anpassung des Vertrages gemäß $\$ 313$ BGB zuzustimmen. ${ }^{60}$ Gleiches gilt regelmäßig, wenn der Bauunternehmer infolge der Covid-19-Pandemie Insolvenz anmeldet. ${ }^{61}$ Nach einer solchen außerordentlichen Kündigung kann der Unternehmer dann eine Teilvergütung für den bereits erbrachten Leistungsteil verlangen ( $\$ 648 \mathrm{a}$ Abs. 5 BGB).

b) Rücktritt des Bestellers ( $\$ 323 \mathrm{BGB})$

Möglich bleibt wie im Kaufrecht ein Rücktritt des Bestellers nach $₫ 323$ Abs. 1 BGB, wenn der Fälligkeitstermin für die Leistung des Werkunternehmers bereits überschritten ist und der Besteller dem Unternehmer eine angemessene Nachfrist gesetzt hat, die fruchtlos abgelaufen ist. Die Nachfristsetzung ist wie im Kaufrecht gemäß $₫ 323$ Abs. 2 Nr. 2 BGB entbehrlich, wenn ein sogenanntes „relatives Fixgeschäft“ vorliegt. Ein solches Rücktrittsrecht wird man in aller Regel bei Beförderungsverträgen annehmen können, die für einen bestimmten Termin abgeschlossen wurden, etwa ein Zug-, Flug- oder Busticket für eine bestimmte Verbindung. Hier liegt zwar nach zutreffender Auffassung kein absolutes Fixgeschäft vor, weil die Beförderungsleistung als solche auch zu einem späteren Termin noch erbracht werden kann; ${ }^{62}$ regelmäßig wird der Kunde an ihr allerdings kein Interesse mehr haben, weil das Ticket für einen konkreten, zeit-

60 W. Voit, in: H. G. Bamberger et al. (Hrsg.), Beck'scher Online-Kommentar zum BGB (Fn. 11), 1.2.2019, $\$ 648$ a Rn. 4.

61 Näher H. Reiter, in: beck-online.Großkommentar zum Zivilrecht (Fn.14), 1.4.2020, \$ 648 a Rn. 26 ff.

62 BGH NJW 2009, 2743 (zur Flugreise). 
lich gebundenen Aufenthalt am Zielort gebucht war. Unbeschadet der Stornobedingungen des jeweiligen Reiseunternehmens ist daher in diesen Fällen ein Rücktritt nach $\ 323$ Abs. 2 Nr. 2 BGB möglich. Ähnlich dürfte es bei Werkverträgen über die Erstellung zeitgebundener temporärer Bauwerke (z.B. eines Messestandes) liegen, wenn absehbar ist, dass der Bauunternehmer diese aus pandemiebedingten Gründen nicht rechtzeitig fertigstellen kann (solange die Messe überhaupt noch stattfinden konnte; andernfalls liegt zugleich eine Störung auf Seiten des Bestellers vor, s. dazu unten E.II).

Ein relatives Fixgeschäft dürfte auch ein Vertrag zwischen einem Veranstalter und einem Künstler oder Ensemble über ein Konzert oder eine sonstige Aufführung ${ }^{63} \mathrm{zu}$ einem bestimmten Termin an einem bestimmten Ort sein, weil eine spätere Nachholung der Veranstaltung durchaus im naturgesetzlichen Sinne möglich ist; ${ }^{64}$ typischerweise ist eine Nachfristsetzung hierfür allerdings nicht sinnvoll, sondern eher das freie Rücktrittsrecht des Veranstalters, wie es sich aus $\$ 323$ Abs. 2 Nr. 2 BGB ergibt. Freilich setzt dieses voraus, dass die Leistungserbringung an sich möglich gewesen wäre und die Störung ausschließlich in der Sphäre des Künstlers lag. Scheitert die Veranstaltung schon daran, dass am vereinbarten Ort zur vereinbarten Zeit jegliche Veranstaltung untersagt war, liegt eine Störung auf Seiten des Bestellers vor (s. dazu E.II).

Folge eines solchen Rücktritts ist, dass der Unternehmer von seiner Leistungspflicht frei wird, aber zugleich auch seinen Vergütungsanspruch vollständig verliert; etwa bereits bezahlte Vorschüsse sind nach $\$ 346$ Abs. 1 BGB zu erstatten. Nach der Konzeption des Gesetzes gilt das auch dann, wenn er mit der Werkleistung bereits begonnen und Aufwendungen getätigt hatte, sofern die erbrachten Teilleistungen für den Kunden nicht von Interesse sind ( $\$ 323$ Abs. 5 S. 1 BGB).

\section{c) Absolute Fixgeschäfte ( $\$ \mathbb{S} 275$ Abs. 1, 326 BGB)}

Bei Werkverträgen sind auch absolute Fixgeschäfte nicht selten, bei denen die Leistung ihrer Natur nach nur zu einem bestimmten Zeitpunkt erbracht werden kann. Soll etwa ein Fotograf im Rahmen eines Werkver-

63 Hierbei handelt es sich i.d.R. um einen Werkvertrag, OLG Köln, MDR 1995, 133; OLG München NJW-RR 2005, 616; Spenner/Estner, Veranstaltungsabsagen (Fn. 59), S. 854.

64 Für absolutes Fixgeschäft allerdings wohl Spenner/Estner, Veranstaltungsabsagen (Fn. 59), S. 853. 
trags von einem bestimmten Ereignis Aufnahmen anfertigen, so wird die Leistung in dem Moment unmöglich, in welchem dieses Ereignis vorbei ist. Kann der Fotograf also pandemiebedingt ein (trotz der Corona-bedingten Einschränkungen stattfindendes) Ereignis nicht dokumentieren, so ist die Leistung insgesamt tatsächlich unmöglich im Sinne von $\mathbb{2 7 5}$ Abs. 1 BGB; der Kunde schuldet nach $₫ 326$ Abs. 1 BGB kein Honorar und kann eine bereits geleistete Vorauszahlung nach $\$ 326$ Abs. 4 BGB zurückverlangen.

d) Leistungsverweigerung aus persönlichen Gründen ( $\$ 275$ Abs. 3 BGB)

Bei Werkverträgen sind schließlich auch Fälle denkbar, in denen der Werkunternehmer seine Leistung nach $\$ 275$ Abs. 3 BGB verweigern darf, weil sie ihm aus persönlichen Gründen unzumutbar ist. Das gilt nur für Fälle, in denen die Werkleistung tatsächlich höchstpersönlicher Natur ist, also nach dem Inhalt des Vertrages nicht auch von einem Dritten erbracht werden könnte. So kann etwa ein Solokünstler seinen gebuchten Auftritt nach $\$ 275$ Abs. 3 BGB verweigern, wenn er zu einer Risikogruppe gehört und deswegen wegen der mit einer Erkrankung verbundenen Gefahr für Leib und Leben keinerlei Ansteckungsrisiko in Kauf nehmen möchte. ${ }^{65}$ Das dürfte allerdings nicht gelten, wenn er zur Kinderbetreuung zu Hause bleibt, weil diese grundsätzlich delegierbar ist; inwieweit er die dafür erforderlichen Kosten übernehmen muss, bestimmt sich nach $\mathbb{} 275$ Abs. 2 BGB. ${ }^{66}$ Mit der Geltendmachung der Einrede aus $₫ 275$ Abs. 3 BGB verliert der Künstler allerdings zugleich seinen Honoraranspruch ( $\$ 326$ Abs. 1 BGB) und muss eine etwa erbrachte Vorleistung nach $\$ 326$ Abs. 4 BGB erstatten.

e) Leistungsverweigerung wegen unverhältnismäßigen Aufwandes ( $\mathbb{2 7 5}$ Abs. 2 BGB)

Eine weitere denkbare Störung auf Seiten des Unternehmers ist eine Steigerung des Leistungsaufwands, etwa weil Material in der Beschaffung pandemiebedingt teurer wird. Auch hier ist zunächst zu prüfen, ob das Risiko der Kostenerhöhung für das Material vertraglich auf den Besteller übertra-

65 Lorenz (Fn. 12), \1 Rn. 25; Riehm (Fn. 20), \275 Rn. 271.1.

66 Dazu sogleich. 
gen wurde oder beim Unternehmer liegt. Ist ohnehin eine Abrechnung nach dem tatsächlichen Materialaufwand vereinbart, so stellt sich die Frage eines Leistungsverweigerungsrechts des Unternehmers nicht, weil er die erhöhten Kosten schlicht an den Käufer durchreichen kann; lediglich aus dessen Sicht kommt eine Vertragsanpassung oder sogar ein Rücktritt vom Vertrag wegen Wegfalls der Geschäftsgrundlage in Betracht, wenn exorbitant gestiegene Kosten dazu führen, dass aus seiner Sicht das Äquivalenzverhältnis von Leistung und Gegenleistung nicht mehr gewahrt ist. Trägt allerdings der Unternehmer das Risiko einer Kostensteigerung beim Material (z.B. bei einem Pauschalpreisvertrag), so ist der Maßstab für die geschuldeten Mehraufwendungen des Unternehmers $\$ 275$ Abs. 2 BGB. Bei allgemein steigenden Materialkosten wird allerdings typischerweise auch das Leistungsinteresse des Bestellers parallel zu den steigenden Kosten des Unternehmers wachsen, sodass $\$ 275$ Abs. 2 BGB nicht einschlägig ist. ${ }^{67}$ Auch diese Fälle sind allenfalls als Äquivalenzstörungen über $\$ 313$ Abs. 1 BGB zu lösen (bzw. ggfs. vorrangig über $\$ 2$ Abs. 7 VOB/B), wobei zu berücksichtigen ist, dass der Unternehmer das Risiko üblicher Kostensteigerungen im Pauschalpreisvertrag gerade vertraglich übernommen hatte, sodass ein Wegfall der Geschäftsgrundlage allenfalls in Betracht kommt, wenn die Kostensteigerungen so exorbitant sind, dass sie jenseits der erwartbaren Preisschwankungen liegen und ein Festhalten am unveränderten Vertrag dem Unternehmer schlechterdings nicht mehr zugemutet werden kann. ${ }^{68}$

Anders liegt es in dem oben zitierten Fall, dass der Unternehmer wegen des pandemiebedingten Ausfalls von Kinderbetreuungseinrichtungen seine Leistung nur erbringen kann, wenn er gegen zusätzliche Kosten eine anderweitige Betreuung sicherstellen kann. Hier steht dem erhöhten Leistungsaufwand des Unternehmers kein parallel erhöhtes Leistungsinteresse des Bestellers gegenüber, sodass eine Berufung auf $\$ 275$ Abs. 2 BGB durchaus möglich erscheint, wenn die Mehrkosten für die anderweitige Betreuung dazu führen, dass der Gesamtaufwand des Künstlers völlig außer Verhältnis zum Leistungsinteresse des Auftraggebers steht. Dieses Leistungsinteresse kann allerdings weit über das vereinbarte Honorar hinausgehen, weil es auch sonstige zwecklos gewordene Aufwendungen des Auftraggebers $(\mathbb{2} 284 \mathrm{BGB})$ und sogar die Erstattung etwaiger Eintrittsgelder umfasst,

67 S. dazu oben D.I.2.

68 S. etwa BGH WM 1964, 1253 zu gestiegenen Lohnkosten beim Pauschalpreisvertrag. 
falls der Auftritt des Künstlers nicht angemessen substituiert werden kann. ${ }^{69}$

\section{Störungen auf Seiten des Bestellers}

Beim Werkvertrag können Störungen auch den Besteller betreffen, z.B. wenn für sein Baugrundstück ein Betretungsverbot angeordnet oder die Veranstaltung eines Konzerts mit den engagierten Solokünstlern, für die vielleicht auch ein Caterer beauftragt wurde, als Großveranstaltung behördlich verboten wird: Diese Verbote treffen nicht den Unternehmer selbst, der seine Leistung (Bauen, Konzert oder Catering) an sich noch erbringen könnte und dürfte, sondern den jeweiligen Besteller, der deswegen die Annahme der Leistung verweigern muss.

Hier sieht zwar $₫ 642$ BGB einen Anspruch des Werkunternehmers auf angemessene Entschädigung vor, wenn der Besteller eine notwendige Mitwirkungshandlung unterlässt und dadurch in Annahmeverzug gerät. Allerdings besteht dieser Anspruch nach der jüngeren Rechtsprechung des BGH nur für solche Hindernisse auf Seiten des Bestellers, die von ihm beeinflusst werden können. ${ }^{70}$ Das kann man bei der Covid-19-Pandemie mit guten Gründen bestreiten ${ }^{71}$ - wenngleich der von $\$ 642$ Abs. 1 BGB geforderte Annahmeverzug kein Verschulden des Bestellers voraussetzt. ${ }^{72}$ Gleichwohl dürfte bei diesen Fällen höherer Gewalt eine Entschädigung nach $₫ 642$ Abs. 1 BGB ausgeschlossen sein. Richtigerweise sollte das auch dann gelten, wenn das Hindernis für den Unternehmer darin besteht, dass pandemiebedingt die Leistung eines Vorunternehmers nicht abgeschlossen werden konnte. ${ }^{73}$ Jeweils ist allerdings zu fordern, dass der Besteller alles in seiner Macht Stehende unternommen hat, um die behördliche Untersagung bzw. die damit verbundenen Störungen des Ablaufs zu vermeiden; andernfalls sind das Unterlassen einer Mitwirkungshandlung und ein Annahmeverzug des Bestellers i.S.v. $\$ 642$ Abs. 1 BGB zu bejahen, sodass ein

69 Zum Erstattungsanspruch der Konzertbesucher s. Spenner/Estner, Veranstaltungsabsagen (Fn. 59), S. 853; Weller/Lieberknecht/Habrich, Virulente Leistungsstörungen (Fn. 1), S. 1020; zur nunmehr eingeführten „Gutscheinlösung“ s. Lorenz (Fn. 12), $\mathbb{1} 1 \mathrm{Rn} .27 \mathrm{ff}$.

70 BGH NJW 2017, 2025 Rn. 19.

71 Offener gegenüber einer Anwendung des $\$ 642$ BGB in diesen Fällen offenbar Weiser, Coronavirus (Fn. 55), S. 206.

72 Voit (Fn. 60), \$ 642 Rn. 12.

73 A.A. wohl Fuchs/Dreher, Corona-Pandemie (Fn. 58), S. 202. 
Ausgleichsanspruch besteht. Der Annahmeverzug setzt in diesem Fall allerdings voraus, dass der Unternehmer leisten darf sowie zur Leistung tatsächlich bereit und in der Lage ist. ${ }^{74}$ Liegt also zugleich ein Fall der Verhinderung des Unternehmers vor (s. oben F.I), gehen die Rechtsfolgen der (vorübergehenden oder endgültigen) Unmöglichkeit vor, sodass ein Anspruch aus $\$ 642 \mathrm{BGB}$ ebenfalls ausscheidet.

Bei absoluten Fixgeschäften tritt mit Zeitablauf Unmöglichkeit der Leistung ein; ist der Besteller zu diesem Zeitpunkt bereits in Annahmeverzug - etwa ein Veranstalter, der das Konzert abgesagt hat -, bleibt gem. $\$ 326$ Abs. 2 S. 1 Var. 2. BGB der Anspruch des Unternehmers auf die Gegenleistung erhalten (s. auch $₫ 644$ Abs. 1 S. 2 BGB). ${ }^{75}$ Ein wörtliches Angebot gem. $\$ 295$ BGB ist hier grundsätzlich entbehrlich, wenn der Besteller durch die Absage unmissverständlich deutlich gemacht hat, dass er die Werkleistung in keinem Fall annehmen wird. ${ }^{76}$ Hier differenziert die Rechtsprechung bisher nicht danach, ob der Grund für den Annahmeverzug des Bestellers von diesem beeinflusst werden konnte. ${ }^{77}$ Teilweise wird allerdings vertreten, dass ein behördliches Veranstaltungsverbot zugleich die Unmöglichkeit der Werkleistung des Künstlers bzw. Ensembles bewirke, was einem Annahmeverzugshonorar entgegenstehe. ${ }^{78}$ Das überzeugt jedoch nicht uneingeschränkt: Die geschuldete Leistung des Künstlers besteht nur darin, die vereinbarte Aufführung an dem vereinbarten Ort zu präsentieren. Die Bereitstellung des Ortes und die „Verschaffung“ von Publikum sind die Obliegenheit des Veranstalters, nicht Teil der Leistungspflicht des Künstlers. Daher ist nach dem exakten Inhalt der behördlichen Anordnung zu differenzieren: Ist die konkrete Aufführung selbst verboten (etwa weil in einem Chor oder Orchester der erforderliche Mindestabstand nicht eingehalten werden kann), liegt tatsächlich Unmöglichkeit der Werkleistung des Chors oder Orchesters vor, sodass kein Annahmeverzugshonorar geschuldet ist. Ist allerdings die Darbietung selbst mit den behördlichen Schutzanordnungen vereinbar, darf nur der Darbietungsort nicht für Publikum geöffnet werden, liegt dies im Risikobereich des Veranstalters und begründet keine Unmöglichkeit der geschuldeten (Werk-)Leistung, sodass der werkvertragliche Honoraranspruch des Künstlers grundsätzlich bestehen bleibt.

74 Voit (Fn. 60), \$642 Rn. 11.

75 Spenner/Estner, Veranstaltungsabsagen (Fn. 59), S. 855.

76 S. Dötterl, in: beck-online.Großkommentar zum Zivilrecht (Fn. 14), 1.1.2020, $₫ 295 \mathrm{Rn} .29 \mathrm{ff}$.

77 Dötterl (Fn. 76), $\$ 293$ Rn. 92; Voit (Fn. 60), $\$ 644$ Rn. 14.

78 Spenner/Estner, Veranstaltungsabsagen (Fn. 59), S. 855. 
Eine andere Lösung kann sich allenfalls aus $₫ 313$ Abs. 1 BGB ergeben, wenn man pandemiebedingte Störungen als nicht von der Risikoverteilung der $\$ \$ 326$ Abs. 2 S. 1 Var. 2, 644 Abs. 1 S. 2 BGB erfasst ansieht.

\section{F. Leistungsschwierigkeiten beim Dienstvertrag}

Abschließend sei noch ein Blick auf Corona-bedingte Leistungsstörungen beim Dienstvertrag geworfen. Die Ergebnisse stimmen allerdings im Wesentlichen mit denen beim Werkvertrag überein. Beispiele für Dienstverträge in diesem Zusammenhang sind Verträge mit mitwirkenden Künstlern im Orchester oder in einer Band für Konzerte (anders als der Vertrag zwischen Veranstalter und Orchester bzw. Band, der typischerweise ein Werkvertrag sein wird), im Event-Bereich zudem auch Verträge mit Bewachungsunternehmen und anderen Dienstleistern. Hinzu kommen klassische Beispiele von freien Dienstverträgen wie solche über Sport-, Musikoder Nachhilfeunterricht, sofern dieser nicht über digitale Medien substituiert werden kann.

\section{Störungen auf Seiten des Dienstverpflichteten}

Auch hier können die Leistungsstörungen auf beiden Seiten eintreten: Der Dienstverpflichtete kann durch Krankheit oder behördliche Anordnung an seiner Leistungserbringung gehindert werden; dann liegt ein Fall der (ggfs. vorübergehenden) Unmöglichkeit vor, die im Falle nicht nachholbarer Dienstleistungen (also absoluter Fixgeschäfte) zur endgültigen Unmöglichkeit wird. Dementsprechend ist der Erfüllungsanspruch des Dienstberechtigten analog bzw. gemäß $₫ 275$ Abs. 1 BGB vorübergehend suspendiert oder vollständig ausgeschlossen. Die Gegenleistungspflicht des Auftraggebers ist ihrerseits dann nach $₫ 320$ BGB vorübergehend suspendiert bzw. bei absoluten Fixschulden nach $\$ 326$ Abs. 1 BGB ausgeschlossen. Gleiches gilt bei höchstpersönlichen Leistungspflichten, wenn die Dienstleistung für den Dienstverpflichteten aus persönlichen Gründen unzumutbar i.S.v. $\$ 275$ Abs. 3 BGB ist (z.B. nicht delegierbarer Betreuungsbedarf, schwere Erkrankung eines nahen Angehörigen o.ä.). Sofern keine absolute Fixschuld vorliegt, bleibt auch hier der Vertrag faktisch in der Schwebe, bis eine Seite ihn nach $₫ 626$ BGB außerordentlich kündigt, weil sein Inter- 
esse an der Leistung weggefallen ist oder ihm ein weiteres Zuwarten bis zu einer möglichen Nachholung der Leistung nicht mehr zumutbar ist. ${ }^{79}$

\section{Störungen auf Seiten des Dienstberechtigten}

Tritt die Störung auf Seiten des Dienstberechtigten ein, obwohl der Dienstverpflichtete seine Leistung an sich erbringen könnte (z.B. der Veranstalter muss das Konzert, für das ein Security-Unternehmen beauftragt war, wegen eines generellen Veranstaltungsverbots absagen), so kommt dieser in Annahmeverzug und schuldet nach $₫ 615$ BGB gleichwohl die Vergütung. Anders als bei $\$ 642 \mathrm{BGB}$ hat die Rechtsprechung zu $\ 615$ BGB bisher keine Einschränkungen in dem Sinne vorgenommen, dass die Nichtannahme der Dienstleistung nicht auf höherer Gewalt beruhen dürfe; nach h.M. gilt $\$ 615$ BGB unabhängig davon, ob der Gläubiger die Dienste nicht annehmen kann oder nicht annehmen will. ${ }^{80}$ Allerdings sind derartige Fälle, soweit ersichtlich, bisher auch noch nicht zu Gericht gelangt. Soweit das BAG den Annahmeverzug ausschließt, weil dem Arbeitgeber „nach Treu und Glauben unter Berücksichtigung der Gepflogenheiten des Arbeitslebens die Annahme der Arbeitsleistung unzumutbar ist" ${ }^{81}$ betrifft diese Rechtsprechung ausschließlich Fälle, in denen der Arbeitnehmer durch sein Verhalten (z.B. Straftaten gegen den Arbeitgeber) diesem die Annahme der Arbeitsleistung unzumutbar gemacht hat. Objektive Unzumutbarkeitsgründe oder Unmöglichkeitsgründe wie die hier vorliegenden behördlichen Anordnungen sind davon nicht umfasst.

Die Anwendung des $₫ 615$ BGB setzt allerdings voraus, dass der Dienstverpflichtete zur Leistung der Dienste tatsächlich bereit und in der Lage ist. ${ }^{82}$ Liegt also zugleich ein Fall der Verhinderung des Dienstverpflichteten vor (s. oben F.I), gehen die Rechtsfolgen der (vorübergehenden oder endgültigen) Unmöglichkeit vor, sodass dem Dienstverpflichteten kein Annahmeverzugshonorar zusteht.

79 S. auch Bacher, Corona-Pandemie (Fn.7), S. 519: Kündigungsrecht nach $₫ 314$ BGB.

80 M. Bieder, in: beck-online.Großkommentar zum Zivilrecht (Fn. 14), 1.2.2020, $₫ 615$ Rn. 15; R. Richardi/P. Fischinger, in: J. von Staudinger (Hrsg.), Kommentar zum Bürgerlichen Gesetzbuch (Fn. 22), 2016, \$615 Rn. 95.

81 BAG NJW 2016, 1977; NZA 2014, 1082; Dötterl (Fn. 76), \$ 293 Rn. 94 ff.

82 A. Baumgärtner, in: H. G. Bamberger et al. (Hrsg.), Beck'scher Online-Kommentar zum BGB (Fn. 11), 1.2.2020, $\$ 615$ Rn. 12. 
Dementsprechend haben auch im Wege eines freien Dienstvertrags beschäftigte Orchestermusiker, die an einer abgesagten Aufführung hätten mitwirken sollen, nach $\$ 615$ BGB grundsätzlich Anspruch auf das entgangene Honorar (abzüglich ersparter Aufwendungen, etwa für Fahrtkosten,

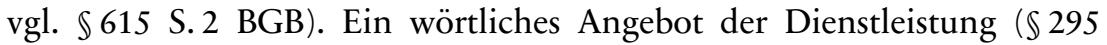
BGB) ist hierfür nicht erforderlich, weil der Konzertveranstalter durch seine Absage zum Ausdruck bringt, dass er die Dienste des Musikers für die geplante Aufführung unter keinen Umständen anzunehmen bereit ist. ${ }^{83}$ Auch beim Engagement von Künstlern (etwa zur Mitwirkung an einer Orchesteraufführung) wird allerdings vertreten, dass ein behördliches Veranstaltungsverbot die Unmöglichkeit der Dienstleistung bewirke. ${ }^{84}$ Auch hier wird man wiederum - wie beim Werkvertrag (oben E.II) - nach dem genauen Inhalt der behördlichen Anordnung differenzieren müssen.

\section{G. Fazit}

Betrachtet man die vertragstypenübergreifenden Rechtsfolgen der typischen Corona-bedingten Leistungsstörungen, so lässt sich folgendes Bild festhalten:

1. Soweit die Parteien - etwa in einer Force Majeure-Klausel oder in einer MAC-Klausel - das Risiko von Leistungsstörungen infolge höherer Gewalt explizit unter sich verteilt haben, ist im Wege der Auslegung der jeweiligen Klausel zu ermitteln, ob sie auch das Pandemierisiko umfasst. Bei Force Majeure-Klauseln wird das in der Regel der Fall sein, wenn der Vertrag vor Bekanntwerden der Pandemie abgeschlossen wurde. Soweit diese Klausel wirksam vereinbart wurde, gelten vorrangig die darin festgelegten Rechtsfolgen.

2. Geldschuldner geraten - vorbehaltlich des Moratoriums gem. Art. 240 $\$ 1$ EGBGB - auch bei Corona-bedingten Zahlungsschwierigkeiten in Verzug („Geld hat man zu haben“). Abhilfe kann hier allenfalls durch eine Vertragsanpassung oder -aufösung nach den Grundsätzen vom Wegfall der Geschäftsgrundlage gem. $\$ 313$ BGB geschaffen werden.

3. Bei Störungen auf Seiten des Leistungsschuldners (Verkäufers, Werkunternehmers, Dienstverpflichteten) bestimmen sich die Grenzen der

83 S. dazu BGH NJW 2001, 287 (288); Dötterl (Fn. 76), \$295 Rn. 30.

84 Spenner/Estner, Veranstaltungsabsagen (Fn. 59), S. 855; s. zur Parallelproblematik beim Werkvertrag oben E.II. 
jeweiligen Leistungspflicht nach $\$ 275$ Abs. 1 bis 3 BGB sowie ggfs. nach einer Vertragsanpassung gem. $\$ 313$ BGB.

a. Soweit diese Grenzen noch nicht erreicht sind, bleibt er zur Leistung verpflichtet und schuldet bei Nichtleistung Verzögerungsschaden sowie ggfs. Schadensersatz statt der Leistung gem. $\$ \mathbb{\$} 280$ Abs. 1, Abs. 3, 281 BGB; zudem ist der Gläubiger (Käufer, Besteller, Dienstberechtigte) regelmäßig zum Rücktritt bzw. zur außerordentlichen Kündigung berechtigt. Bei einem absoluten Fixgeschäft hat der Gläubiger in diesem Fall unmittelbar einen Anspruch auf Schadensersatz statt der Leistung gem. $\$ \mathbb{\$} 280$ Abs. 1, Abs. 3, 283 BGB.

b. Sind die Grenzen der Leistungspflicht gem. $\$ 275$ BGB überschritten, liegt typischerweise ein Fall der vorübergehenden Unmöglichkeit, Leistungserschwerung oder persönlichen Unzumutbarkeit vor. Dies führt im praktischen Ergebnis dazu, dass die beiderseitigen Leistungspflichten analog $\$ 275$ BGB bzw. gem. $\int \$ 320 \mathrm{f}$. BGB für die Dauer des Leistungshindernisses suspendiert sind; der Vertrag bleibt gewissermaßen „in der Schwebe“. Der Gläubiger kann diese Schwebelage allerdings durch einen Rücktritt gem. $\$ 323$ BGB beenden; dieses Rücktrittsrecht ist von einem Verschulden des Schuldners unabhängig und setzt entweder eine Fristsetzung gem. $₫ 323$ Abs. 1 BGB oder einen Entbehrlichkeitsgrund nach $\$ 323$ Abs. 2 BGB voraus. Bei absoluten Fixgeschäften tritt mit Ablauf des Leistungszeitraums dauernde Unmöglichkeit ein, sodass die Gegenleistungspflicht unmittelbar gem. $\$ 326$ Abs. 1 BGB entfällt und eine ggfs. schon erbrachte Gegenleistung gem. $\$ 326$ Abs. 4 BGB zu erstatten ist.

4. Bei Störungen auf Seiten des Leistungsgläubigers tritt - wenn der Schuldner an sich leisten dürfte und könnte - Annahmeverzug ein. Im Dienstvertrag kann der Dienstverpflichtete dann ein Annahmeverzugshonorar gem. $\$ 615$ BGB verlangen, selbst wenn der Grund für die Annahmeverweigerung sich aus Sicht des Dienstberechtigten als höhere Gewalt darstellt. Beim Werkvertrag schließt die Rechtsprechung dagegen die Anwendung des $₫ 642$ BGB zugunsten des Unternehmers aus.

5. Alle vorstehend genannten leistungsstörungsrechtlichen Folgen stehen unter dem Vorbehalt einer möglichen Vertragsanpassung oder -auflösung wegen Wegfalls der Geschäftsgrundlage ( $\$ 313$ BGB). Nur diese Regelung führt potenziell zu einer gleichmäßigen Verteilung des (universellen) Pandemierisikos zwischen den Parteien anstelle einer „Allesoder-Nichts"-Lösung, wie sie das allgemeine Leistungsstörungsrecht typischerweise bewirkt. 
6. Wegen der mit der überlagernden Anwendung des $₫ 313$ BGB verbundenen Unsicherheiten empfiehlt es sich dringend, bei Corona-bedingten Leistungsstörungen als ersten Schritt Verhandlungen zwischen den Vertragspartnern aufzunehmen, um eine konsensuale, dem Einzelfall angemessene Verteilung der pandemiebedingten Einbußen zwischen den Parteien zu erzielen. ${ }^{85}$

85 Ebenso die Empfehlung von C. Armbrüster, Corona - Zeit zum Nachverhandeln!, NJW-aktuell 16/2020, S. 3 ff. 
